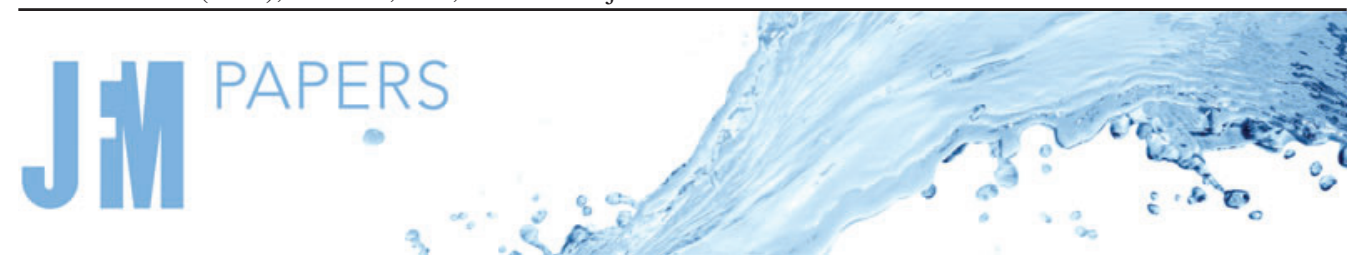

\title{
Free-stream coherent structures in parallel compressible boundary-layer flows at subsonic and moderate supersonic Mach numbers
}

\author{
Eleanor C. Johnstone ${ }^{1, \dagger}$ and Philip Hall ${ }^{2}$ \\ ${ }^{1}$ Department of Mathematics, University of Manchester, Manchester M13 9PL, UK \\ ${ }^{2}$ School of Mathematics, Monash University, Melbourne, VIC 3800, Australia
}

(Received 6 November 2020; revised 31 March 2021; accepted 4 July 2021)

As a first step towards the description of coherent structures in compressible shear flows, we present an asymptotic description of nonlinear travelling-wave solutions of the Navier-Stokes equations in the compressible asymptotic suction boundary layer (ASBL). We consider free-stream Mach numbers $M_{\infty}$ in the subsonic and moderate supersonic regime so that $0 \leqslant M_{\infty} \leqslant 2$. We extend the large-Reynolds-number asymptotic theory of Deguchi \& Hall (J. Fluid Mech., vol. 752, 2014, pp. 602-625) describing 'free-stream' coherent structures in incompressible ASBL flow to describe a nonlinear interaction in a thin layer situated just below the free stream. Crucially, the nonlinear interaction equations for the velocity field in this layer are identical to those obtained in the incompressible problem, and thus the asymptotic analysis supporting free-stream coherent structures in compressible ASBL is easily deduced from its incompressible counterpart. The nonlinear interaction produces streaky disturbances to both the velocity and temperature fields, which can grow exponentially towards the wall. We complete the description of the growth of the velocity and thermal streaks throughout the flow by solving the compressible boundary-region equations numerically. We show that the velocity and thermal streaks obtain their maximum amplitude in the unperturbed boundary layer. Increasing the free-stream Mach number enhances the thermal streaks and suppresses the velocity streaks, whereas varying the Prandtl number suppresses the velocity streaks, and can either enhance or suppress the thermal streaks depending on whether the flow is in the subsonic or moderate supersonic regime. Such nonlinear equilibrium states have been implicated in shear transition in incompressible flows; therefore, our results indicate that a similar mechanism may also be present in compressible flows.

Key words: compressible boundary layers, transition to turbulence, nonlinear instability

$†$ Email address for correspondence: eleanor.johnstone@manchester.ac.uk

(C) The Author(s), 2021. Published by Cambridge University Press. This is an Open Access article, distributed under the terms of the Creative Commons Attribution licence (https://creativecommons. org/licenses/by/4.0/), which permits unrestricted re-use, distribution, and reproduction in any medium, provided the original work is properly cited. 


\section{Introduction}

It has been known since Kline et al. (1967) that transitional and turbulent flows exhibit clear structure within the boundary layer in the form of vortical structures coupled to high- and low-speed streaks in the plane perpendicular to the unperturbed flow. Recent understanding of these structures has been aided by the identification of three-dimensional, nonlinear invariant solutions of the Navier-Stokes equations which may take the form of equilibria, periodic orbits or travelling-wave solutions. These states, now commonly known as exact coherent structures, have been found in a wide range of canonical shear flows where the key parameter governing the dynamics is the Reynolds number; see, for e.g. Faisst \& Eckhardt (2003), Waleffe (2001, 2003), Wedin \& Kerswell (2004) and Wang, Gibson \& Waleffe (2007). The study of exact coherent structures in two-parameter space has previously only been conducted in the context of stably stratified flows (Eaves \& Caulfield 2015; Deguchi 2017; Lucas \& Caulfield 2017; Lucas, Caulfield \& Kerswell 2017; Olvera \& Kerswell 2017), where it is shown that the Prandtl number plays a key role in the structure of the states found (Langham, Eaves \& Kerswell 2020).

The present work is confined to a special type of coherent structure in asymptotic suction boundary-layer (ASBL) flow, in which a parallel, streamwise-invariant basic flow is maintained via constant suction far from the leading edge. In the incompressible case, Hocking (1975) showed that the flow is linearly stable up to a Reynolds number of 54 370; Fransson \& Alfredsson (2003) subsequently showed experimentally that transition could occur at much lower Reynolds numbers. It has been very recently shown that it is possible to experimentally realise a turbulent ASBL (Ferro, Fallenius \& Fransson 2021). Several three-dimensional, fully nonlinear invariant solutions of the Navier-Stokes equations have been identified in incompressible ASBL flow. Periodic-orbit-type solutions have been obtained by Kreilos et al. (2013) and Khapko et al. (2013) via edge tracking. Travelling-wave-type solutions have also been identified in the ASBL by Deguchi \& Hall (2014), who found structures localised in the wall-normal direction but periodic in the streamwise and spanwise directions, and by Kreilos, Gibson \& Schneider (2016), who found spanwise-localised travelling-wave solutions. In both cases, two types of solution were found: a 'wall mode' coherent structure with the streaks and vortex structure concentrated near the wall region; and a 'free-stream' coherent structure with the streak flow still mainly concentrated in the near-wall region but with the vortical structure residing in the free stream.

Deguchi \& Hall (2014) showed that the spanwise-periodic wall modes could be described by high-Reynolds-number vortex-wave interaction theory (Hall \& Smith 1991; Hall \& Sherwin 2010), in which forcing in the critical layer of the wave drives a roll flow which produces a streak; the streaky flow is then itself unstable to the wave. This tripartite interaction is also known as a self-sustaining process (Waleffe 1997). Meanwhile, the free-stream coherent structures can be described by a distinct asymptotic theory which relies on the exponential approach of the boundary-layer flow to its free-stream form. A nonlinear interaction between tiny waves, rolls and streaks satisfies the unit-Reynolds-number three-dimensional Navier-Stokes equations within a 'production' layer, which is located at the edge of the free stream and which is of the same depth as the unperturbed boundary layer. The nonlinear production-layer interaction allows a streak disturbance to the velocity field to grow exponentially beneath the production layer. An analysis of the induced roll-streak flow shows that the streak obtains its maximum size in the near-wall boundary layer. This high-Reynolds-number asymptotic framework to describe free-stream coherent structures has subsequently been extended to non-parallel (Deguchi \& Hall 2015, 2018) and unsteady (Johnstone \& Hall 2020) flows. 


\section{Free-stream coherent structures in compressible shear flows}

Free-stream turbulence is known to play a key role in boundary-layer transition (Fransson, Matsubara \& Alfredsson 2005; Fransson \& Shahinfar 2020). It is hypothesised that free-stream coherent structures may play a key role in linking the coherent structures observed in the inner region (near-wall region of intense turbulence production) and outer (large-scale, less active) regions of boundary-layer flow (Deguchi \& Hall 2014). This detail would be particularly relevant in the context of jet acoustics for compressible flows, when disturbances originating in the free stream may be implicated in the high frequency sound often referred to as 'screeching' which is observed in high-speed jet flows (Deguchi \& Hall 2018).

There has been little work, however, into the asymptotic description of coherent structures in the context of compressible flows despite the importance of transitional and turbulent compressible flows to many industrial problems, particularly in the fields of aerospace engineering and acoustics. Past experimental and numerical studies have focused on laminar-turbulent transition in compressible boundary layers in the context of the effect of free-stream vortical disturbances, with particular focus on bypass transition (see, for e.g. Laufer 1954; Kendall 1975; Demetriades 1989; Graziosi \& Brown 2002; Mayer, von Terzi \& Fasel 2011). By extending the incompressible theory of Leib, Wundrow \& Goldstein (1999), Ricco \& Wu (2007) show that free-stream vortical disturbances can induce temperature fluctuations that lead to the formation of 'thermal streaks'; the growth of these streaks is enhanced at larger free-stream Mach numbers, although nonlinear effects were found to inhibit the growth of the streaks (Marensi, Ricco $\& \mathrm{Wu}$ 2017). Short-wavelength free-stream vortical disturbances have also been found to concentrate in the 'edge layer' Wu \& Dong (2016), which is akin to the production layer for free-stream coherent structures described above.

However, the organised streaky structures observed experimentally in incompressible flows have been identified in supersonic compressible flows both experimentally (for a thorough review see Spina, Smits \& Robinson 1994) and numerically (Pirozzoli, Bernardini \& Grasso 2008; Ringuette, Wu \& Martín 2008). The structures found are consistent with the hairpin loop model of wall turbulence, with low-speed, elongated streaks observed in the logarithmic region. Thus there exists compelling evidence for the similarity between compressible and incompressible coherent structures. Indeed, the main effect of compressibility in turbulent shear boundary layers lies in the density fluctuations (Morkovin 1962), and it is generally accepted that for moderate free-stream Mach numbers $M_{\infty} \leqslant 2$, the dynamics of compressible shear boundary layers does not differ greatly from its incompressible counterpart (Spina et al. 1994).

The aim of the present work is to ask: (a) Can we use the high-Reynolds-number asymptotic theory describing free-stream coherent structures in incompressible ASBL flow (Deguchi \& Hall 2014) to describe free-stream coherent structures in compressible ASBL flow in the subsonic and moderate supersonic regimes? And $(b)$, what is the influence of the additional physical parameters, namely the Mach number $M_{\infty}$ and the Prandtl number $\sigma$ ?

Assuming a perfect gas, the basic flow for the compressible ASBL approaches its free-stream form exponentially and thus has the underlying structure required to support the free-stream coherent structures described in Deguchi \& Hall (2014). We find that compressibility effects shift the location of the production layer by a constant proportional to $M_{\infty}^{2}$. However, the key result is that the leading-order equations for the velocity field in the production layer are identical to those for the incompressible problem. Since the asymptotics and numerical solutions agreed well for the incompressible case we expect that this is true for the compressible problem. Moreover, this also represents a significant 


\section{E.C. Johnstone and P. Hall}

computational reduction as the solution of the nonlinear eigenvalue production-layer problem, which was computed by direct numerical simulation by Deguchi \& Hall (2014), can also be used for the compressible problem. However, as discussed at the end of this paper, we expect that this reduction will not hold in general for other compressible regimes at higher free-stream Mach number due to the presence of non-parallel effects and shocks.

The equations for the thermal field in the production layer are passive and driven by the velocity field. This effect arises due to the location of the thin production layer being just below the free stream, where compressibility effects are negligible because the density and viscosity are close to their constant free-stream values. As in the incompressible problem, the nonlinear interaction in the production layer produces a disturbance to the streamwise velocity field (a 'streak') that grows exponentially down towards the wall through interaction with the mean flow. However, the nonlinear interaction also induces a disturbance to the temperature field, a 'thermal streak', which also grows exponentially down towards the wall. The amplitude of the thermal streaks is enhanced as the Mach number is increased whilst the amplitude of the velocity streaks is suppressed. In the subsonic regime the amplitude of the velocity streaks is in general one order of magnitude larger than that of the thermal streaks but the amplitudes become of comparable size in the moderate supersonic regime. At the wall, both the velocity and thermal streaks vanish so as to satisfy the wall boundary conditions. The location where the thermal and velocity streaks attain their maximum amplitude relative to the velocity streak is controlled by the Prandtl number.

The rest of this paper is presented as follows: in $\S 2$, we provide a brief description of free-stream coherent structures in incompressible ASBL flow. We then define the governing equations for compressible ASBL flow in $\S 3$ and find the basic flow in $\S 4$. The production-layer problem is then described in $\S 5$. We present the solution below the production layer and down to the wall in $\S 6$. We then present results for a variety of parameters in $\S 7$ and finally in $\S 8$ we draw some conclusions.

\section{Free-stream coherent structures in incompressible parallel boundary-layer flows}

To provide some context for the discussion of free-stream coherent structures in the compressible ASBL flow, we briefly summarise the results of Deguchi \& Hall (2014) for free-stream coherent structures in incompressible ASBL flow.

Incompressible ASBL flow describes viscous, incompressible flow $\left(u^{*}, v^{*}, w^{*}\right)$ with respect to Cartesian coordinates $\left(x^{*}, y^{*}, z^{*}\right)$, with dynamic viscosity $\mu$ and kinematic viscosity $v$, over a flat plate at $y^{*}=0$. Uniform flow exists in the free stream, so denoting free-stream values by subscript $\infty$, at the free-stream $\left(u^{*}, v^{*}, w^{*}\right)=\left(u_{\infty},-v_{\infty}, 0\right)$. The plate is subject to constant suction, so the velocity at the plate is $\left(u^{*}, v^{*}, w^{*}\right)=$ $\left(0,-v_{\infty}, 0\right)$. Non-dimensionalising the velocity components on the free-stream speed $u_{\infty}$ and the coordinates on the length scale $v / v_{\infty}$, and defining the Reynolds number $R e=u_{\infty} / v_{\infty}$, the basic flow is given by

$$
\left(u_{b}, v_{b}, w_{b}\right)=\left(1-\mathrm{e}^{-y},-\operatorname{Re}^{-1}, 0\right) .
$$

Deguchi \& Hall (2014) showed that, at high Reynolds numbers, the incompressible Navier-Stokes equations allow for nonlinear equilibrium solutions taking the form of a roll-wave-streak interaction propagating in a viscous layer at the outer edge of the boundary layer; this layer is termed the production layer and the solutions are known as free-stream coherent structures. The interaction in the production layer is characterised by nonlinear travelling-wave solutions propagating with wave speed $c$; numerical computations suggest that the asymptotic behaviour of the wave speed is 
$1-c=O\left(R e^{-1}\right)$, so that the wave propagates downstream with almost free-stream speed. The solutions also have streamwise length scales comparable to the spanwise length scales, and the thickness of production layer is comparable to the boundary-layer thickness. Seeking a solution with these scalings, which is periodic in the streamwise and spanwise directions with respective wavenumbers $\alpha$ and $\beta$, shows that the production layer in ASBL flow is located at $y=\ln R e$.

The solution inside the production layer is $U(X, Y, z)=(U, V, W)$, where $(x, y, z)=$ $(X-c t, Y-\ln R e, z), c=1-R e^{-1} c_{1}$, and is determined by numerically solving the full Navier-Stokes equations at unit Reynolds number as a nonlinear eigenvalue problem for the perturbed wave speed $c_{1}$ of the travelling wave:

$$
\begin{gathered}
\left(\left[\boldsymbol{U}+c_{1} \hat{\boldsymbol{i}}\right] \cdot \nabla\right) \boldsymbol{U}=-\nabla P+\nabla^{2} \boldsymbol{U}, \\
\nabla \cdot \boldsymbol{U}=0 .
\end{gathered}
$$

The asymptotic structure of the solution emerging from the lower side of the production layer shows that, below the layer, the disturbance to the streamwise velocity (termed the streak), which occurs as a result of the nonlinear interaction in the production layer, can grow exponentially like $\mathrm{e}^{-Y}$ as $Y \rightarrow-\infty$ while the other velocity components decay. Thus moving beneath the production layer,

$$
\begin{gathered}
u \rightarrow 1-\mathrm{e}^{-y}+\frac{d_{0}}{R e}+\frac{J_{1}}{R e^{\omega_{1}}} \mathrm{e}^{\left(\omega_{1}-1\right) y} \cos (2 \beta z)+\frac{J_{1} K_{1}}{R e^{2 \omega_{1}} 4 \omega_{1}} \mathrm{e}^{\left(2 \omega_{1}-1\right) y}+\cdots, \\
v \rightarrow-\frac{1}{R e}+\frac{K_{1}}{R e^{\omega_{1}+1}} \mathrm{e}^{\omega_{1} y} \cos (2 \beta z)+\cdots, \\
w \rightarrow-\frac{K_{1} \omega_{1}}{2 \beta \operatorname{Re}^{\omega_{1}+1}} \mathrm{e}^{\omega_{1} y} \sin (2 \beta z)+\cdots,
\end{gathered}
$$

where

$$
J_{1}=\frac{K_{1}}{\left(\omega_{1}-1\right)^{2}+\left(\omega_{1}-1\right)-4 \beta^{2}}, \quad \omega_{1}=\frac{-1+\sqrt{1+16 \beta^{2}}}{2} \geqslant 0,
$$

for spanwise wavenumber $\beta$, and where $K_{1}$ is found as part of the numerical solution of the eigenvalue problem in the production layer. These solutions are valid as the wall layer is approached, i.e. when $1 \ll y \ll \ln R e$. The constant of integration $d_{0}$ is found by matching with the numerical solution of the eigenvalue problem (2.2)-(2.3) which was computed for a range of spanwise wavenumbers $\beta$ in Deguchi \& Hall (2014). Thus the term $d_{0} / R e$ is the next order correction to the mean flow due to the nonlinear interaction in the production layer. Therefore in general the streamwise velocity solution is only given up to a constant, however, the correction does not influence the vortex field which is the quantity of interest. By solving for the induced flow throughout the boundary region between the production layer and the wall, Deguchi \& Hall (2014) show that for $\beta<1 / \sqrt{2}$ the streak disturbance grows down to the main part of the boundary layer, before being reduced to zero at the wall to satisfy the boundary conditions.

\section{Governing equations for compressible ASBL flow}

We now consider the compressible counterpart of ASBL flow. Consider a viscous, compressible perfect gas with density, temperature and dynamic viscosity $\rho^{*}, \theta^{*}$ and $\mu^{*}$ respectively, flowing with velocity $\boldsymbol{u}^{*}=\left(u^{*}, v^{*}, w^{*}\right)$ with respect to Cartesian coordinates 


\section{E.C. Johnstone and P. Hall}

$\left(x^{*}, y^{*}, z^{*}\right)$ over an infinitely long flat plate at $y^{*}=0$. Uniform suction exists at the plate boundary so that, denoting free-stream values by subscript $\infty$, the velocity is $\boldsymbol{u}^{*}=\left(0,-v_{\infty}, 0\right)$ at the plate. Meanwhile, a long way from the plate at the free stream, $\boldsymbol{u}^{*} \rightarrow\left(u_{\infty},-v_{\infty}, 0\right)$ and $\left(\rho^{*}, \theta^{*}, \mu^{*}, p^{*}\right) \rightarrow\left(\rho_{\infty}, \theta_{\infty}, \mu_{\infty}, p_{\infty} / \rho_{\infty} u_{\infty}^{2}\right)$. The suction at $y^{*}=0$ does not allow for zero heat transfer over the plate due to the transfer of kinetic energy across it, and therefore we assume the temperature at the plate is fixed so that $\theta^{*}=\theta_{p}$ at $y^{*}=0$.

We non-dimensionalise by scaling the coordinates $\left(x^{*}, y^{*}, z^{*}\right)$ on the velocity-boundarylayer thickness $\delta=\mu_{\infty} / \rho_{\infty} v_{\infty}$, the velocity components $\left(u^{*}, v^{*}, w^{*}\right)$ on $u_{\infty}$, the pressure on $\rho_{\infty} u_{\infty}^{2}$ and the quantities $\rho^{*}, \theta^{*}$ and $\mu^{*}$ on their free-stream values. We define the Reynolds number $R e$ by

$$
R e=u_{\infty} / v_{\infty}
$$

Throughout the analysis that follows, we assume the Reynolds number is large. We also define the following physical constants:

(i) $c_{v}, c_{p}$, are the specific heats at constant volume and constant pressure respectively;

(ii) $\gamma=c_{p} / c_{v}$ is the ratio of specific heats; for air, $\gamma \approx 1.4$;

(iii) $R$ is the molecular gas constant which is approximately $286 \mathrm{~m}^{2} \mathrm{~s}^{-2} \mathrm{~K}^{-1}$ for air;

(iv) $a_{\infty}=\sqrt{\gamma R \theta_{\infty}}$ is the speed of sound in the free stream;

(v) $M_{\infty}=u_{\infty} / a_{\infty}$ is the free-stream Mach number;

(vi) $k$ is the thermal diffusivity of the gas;

(vii) $\sigma=\mu_{\infty} c_{p} / k$ is the Prandtl number which defines the ratio of momentum diffusivity to thermal diffusivity; for air, $\sigma \approx 0.71$.

We consider values of $u_{\infty}$ and $a_{\infty}$ such that we obtain Mach numbers $M_{\infty}$ in the subsonic and moderate supersonic regimes so that $M_{\infty} \leqslant 2$. In the moderate supersonic regime we assume that the plate is sufficiently thin so that shocks are not present. We choose parameters $\gamma$ and $\sigma$ that are appropriate for the ideal gas assumption; in particular, this means that $\sigma<2$, which will become important in the scaling arguments below.

Then, using mixed notation so that $\left(x_{1}, x_{2}, x_{3}\right)$ represents $(x, y, z), \nabla=\left(\partial_{x_{1}}, \partial_{x_{2}}, \partial_{x_{3}}\right)$ and $\boldsymbol{u}=\left(u_{1}, u_{2}, u_{3}\right)$ represents $(u, v, w)$, the Navier-Stokes equations have the form

$$
\begin{aligned}
& \rho \frac{\mathrm{D} u_{i}}{\mathrm{D} t}=-\frac{\partial p}{\partial x_{i}}+\frac{1}{R e}\left\{\frac{\partial}{\partial x_{i}}\left(-\frac{2}{3} \mu \nabla \cdot \boldsymbol{u}\right)+\frac{\partial}{\partial x_{j}}\left(\mu \frac{\partial u_{j}}{\partial x_{i}}\right)+\frac{\partial}{\partial x_{j}}\left(\mu \frac{\partial u_{i}}{\partial x_{j}}\right)\right\} \\
& (i, j=1,2,3) \text {, } \\
& \frac{\partial \rho}{\partial t}+\nabla \cdot(\rho \boldsymbol{u})=0, \\
& \rho \frac{\mathrm{D} \theta}{\mathrm{D} t}=\frac{(\gamma-1) M_{\infty}^{2}}{\operatorname{Re}} \Phi+(\gamma-1) M_{\infty}^{2} \frac{\mathrm{D} p}{\mathrm{D} t}+\frac{1}{\operatorname{Re}} \frac{1}{\sigma} \frac{\partial}{\partial x_{i}}\left(\mu \frac{\partial \theta}{\partial x_{i}}\right), \\
& p=\theta_{\infty} U_{\infty}^{-2} \rho R \theta,
\end{aligned}
$$

where the dissipation function $\Phi$ is defined by

$$
\Phi=\frac{1}{2} \mu e_{i j} e_{i j}-\frac{2}{3} \mu(\nabla \cdot u)^{2},
$$

and $e_{i j}=\partial u_{i} / \partial x_{j}+\partial u_{j} / \partial x_{i}$ is the rate of strain tensor. 


\section{Free-stream coherent structures in compressible shear flows}

We close the equations of motion with a power-law viscosity law, so that after non-dimensionalisation

$$
\mu=\theta^{\zeta}
$$

The index $\zeta=1$ gives the Chapman-Rubesin viscosity law (Chapman \& Rubesin 1949) which is suitable for the subsonic regime; for the moderate supersonic regime a slightly more accurate model has $\zeta=0.76$ (Cebeci 2002). If we were to extend the analysis to higher Mach numbers then a more realistic viscosity model, such as Sutherland's law (Sutherland 1893), would be required.

\section{The basic flow}

We now solve the equations of motion for the basic boundary-layer flow state. The ASBL flow is steady, two-dimensional and independent of $x$. Therefore we seek a boundary-layer solution in the form

$$
\begin{gathered}
(u, v, w, p)=\left(\hat{u}(y), \operatorname{Re}^{-1} \hat{v}(y), 0, \hat{p}(y)\right), \\
(\theta, \rho, \mu)=(\hat{\theta}(y), \hat{\rho}(y), \hat{\mu}(y)),
\end{gathered}
$$

where the scaling for the normal velocity arises from the need to retain viscous effects in the boundary layer. The boundary conditions at the plate and the free stream are given by

$$
\begin{gathered}
(\hat{u}, \hat{v}, \hat{w})=(0,-1,0), \quad \hat{\theta}=\theta_{p} / \theta_{\infty} \quad \text { at } y=0, \\
(\hat{u}, \hat{v}, \hat{w}) \rightarrow(1,-1,0), \quad \hat{p} \rightarrow p_{\infty} / \rho_{\infty} u_{\infty}^{2}, \quad(\hat{\theta}, \hat{\rho}, \hat{\mu}) \rightarrow(1,1,1) \quad \text { as } y \rightarrow \infty .
\end{gathered}
$$

We substitute the expansion (4.1) into the governing equations (3.2)-(3.5) and, assuming that the Reynolds number is large, retain leading-order terms. The $y$-momentum equation from (3.2) with $i=2$ reduces to $\partial \hat{p} / \partial y=0$, which means that the pressure $\hat{p}$ is constant across the boundary layer and equal to its free-stream value of $p_{\infty} / \rho_{\infty} u_{\infty}^{2}$. It follows that the equation of state (3.5) reduces to $\hat{\rho} \hat{\theta}=1$. Then the continuity equation (3.3) reduces to $\partial_{y}(\hat{\rho} \hat{v})=0$; integrating and applying free-stream boundary conditions (4.2) gives $\hat{\rho} \hat{v}=$ -1 across the boundary layer. Thus, $\hat{v}=-\hat{\theta}$, so in particular, the suction condition at the plate gives $\theta_{p} / \theta_{\infty}=1$.

We now use the Dorodnitsyn-Howarth transformation (Dorodnitsyn 1942; Howarth 1948) given by

$$
\xi=\int_{0}^{y} \hat{\rho}\left(y^{\prime}\right) \mathrm{d} y^{\prime},
$$

so that $y$-derivatives $d_{y}$ are replaced by $\hat{\rho}(\xi) d_{\xi}$. The equations of motion then reduce to

$$
\hat{u}^{\prime}+\left(\hat{\theta}^{\zeta-1} \hat{u}^{\prime}\right)^{\prime}=0, \quad \hat{\theta}^{\prime}+\sigma^{-1}\left(\hat{\theta}^{\zeta-1} \hat{\theta}^{\prime}\right)^{\prime}+(\gamma-1) M_{\infty}^{2}\left(\hat{u}^{\prime}\right)^{2}=0
$$

where a prime denotes derivative with respect to $\xi$. In general, these equations must be solved numerically subject to the boundary conditions (4.2). An analytic solution can be 
found in the special case of the Chapman-Rubesin law when $\zeta=1$, and is given by

$$
\begin{gathered}
\hat{u}(\xi)=1-\mathrm{e}^{-\xi}, \quad \hat{\theta}(\xi)=1+\frac{(\gamma-1) M_{\infty}^{2} \sigma}{2(2-\sigma)}\left(\mathrm{e}^{-\sigma \xi}-\mathrm{e}^{-2 \xi}\right), \\
\hat{v}(\xi)=-\hat{\theta}(\xi), \quad \hat{w}(\xi)=0, \quad \hat{p}(\xi)=p_{\infty} / \rho_{\infty} u_{\infty}^{2}, \\
\hat{\rho}(\xi)=(\hat{\theta}(\xi))^{-1}, \quad \hat{\mu}(\xi)=\hat{\theta}(\xi) .
\end{gathered}
$$

For large $\xi$, when the temperature and streamwise velocity are approaching their (non-dimensional) free-stream values of 1 , this analytical basic solution can be used regardless of the index in the viscosity law (3.7). We can invert the Dorodnitsyn-Howarth transformation (4.3) as

$$
y=\int_{0}^{\xi} \hat{\theta}\left(\xi^{\prime}\right) \mathrm{d} \xi^{\prime} .
$$

Thus if $\xi$ is large, then we can approximate the Dorodnitsyn-Howarth variable by

$$
\xi \approx g(y)=y+C_{0} ; \quad C_{0}=\frac{(1-\gamma) M_{\infty}^{2}}{4} .
$$

Consequently, in the free stream, we can write the basic flow in terms of the physical variable $y$. For the interior region we find $\xi=g(y)$ by solving the inversion equation (4.8) numerically.

Thus for large $\xi$, i.e. large $y$, the basic streamwise velocity is given by $\hat{u} \approx 1-\mathrm{e}^{-C_{0}} \mathrm{e}^{-y}$. Thus the streamwise velocity approaches its free-stream form exponentially as a function of distance from the wall. Therefore the free-stream coherent structure theory of Deguchi \& Hall (2014) can be applied. The basic solution for the temperature field also approaches its free-stream form exponentially, with the rate of decay being dependent on the value of the Prandtl number. As discussed above in $\S 3$, gases which provide a good approximation to the ideal gas assumption have Prandtl numbers $\sigma<2$, and therefore the decay of the basic state to its free-stream form will be dominated by the $\exp (-\sigma \xi)$ term in the basic flow $(4.5 a, b)$. Hence, the decay will be slower than that of the streamwise velocity field $\hat{u}$ if $\sigma<1$. Thus the thermal boundary layer is thicker than the velocity boundary layer if $\sigma<1$, and vice versa if $\sigma>1$; this is consistent with laminar boundary-layer theory which suggests that the thickness of the thermal boundary layer $\delta_{\theta}$ scales relative to the thickness of the velocity boundary layer $\delta_{v}$ as $\delta_{\theta} \sim \delta_{v} \sigma^{-1 / 3}$ (Schlichting 1968, p. 307).

\section{The production-layer problem for compressible ASBL flow}

Using the inversion of the Dorodnitsyn-Howarth transformation for large $\xi$ (4.8), at the production layer we obtain $\xi \approx y+C_{0}$, and therefore the solution in the production layer can be expressed in terms of the physical variable $y$. To find the location of the production layer and the scalings of the flow components in the layer, following Deguchi \& Hall (2014), we seek a travelling-wave solution propagating with almost the free-stream speed with wavelengths comparable to the boundary-layer scalings of $\S 4$ so that $\partial_{x}=\partial_{y}=$ $\partial_{z}=O(1)$. Then, if viscosity is to play a role in the interaction, $v=O\left(R e^{-1}\right)$, and by the continuity equation (3.3), $1-u=w=O\left(R e^{-1}\right)$. To retain convective terms in the $x$-momentum equation (3.2) the $\rho\left(\partial_{t}+u \partial_{x}\right)$ term must also be $O\left(R e^{-1}\right)$; this defines the wave dependence in the production layer. The pressure must then be $O\left(R e^{-2}\right)$ to stay in play. 


\section{Free-stream coherent structures in compressible shear flows}

The streamwise component of the velocity field in the production layer must include the basic flow component $(4.5 a, b)$ for matching. For large $\xi$, the basic flow $\hat{u}$ has the form $1-\hat{u}=\exp \left(-y-C_{0}\right)$, therefore, in the production layer, $\mathrm{e}^{-y-C_{0}}=R e^{-1}$. Thus the location of the production layer is given by $y=y_{P L}=\ln R e-C_{0}$; this allows us to define a production-layer variable $Y=y-\ln R e+C_{0}$. The thickness of the production layer must then be $O(1)$ to ensure that the streamwise velocity $u$ can only vary on an $O(1)$ length scale in the production layer.

Thus since $C_{0}<0$ for $\gamma=1.4$, a key feature of the compressible problem is that the location of the production layer where the waves and rolls are concentrated moves further away from the wall as both the Reynolds number and the Mach number increase. Since $C_{0} \propto M_{\infty}^{2}$, it is anticipated that the Mach number may have a strong influence on the hypersonic (large Mach number) production-layer problem; this is discussed further in the conclusion. However, our choice of parameters means that $\left|C_{0}\right| \ll \ln R e$. Therefore the values of $\sigma$ and $M_{\infty}$ do not strongly influence the location of the production layer.

Under the scalings described above, the basic states for the streamwise velocity and temperature $(4.5 a, b)$ in the production layer are given by

$$
\hat{u}=1-\frac{1}{R e} \mathrm{e}^{-Y}, \quad \hat{\theta}=1+\lambda\left(\frac{1}{R e^{\sigma}} \mathrm{e}^{-\sigma Y}-\frac{1}{R e^{2}} \mathrm{e}^{-2 Y}\right),
$$

where

$$
\lambda=\frac{(\gamma-1) M_{\infty}^{2} \sigma}{2(2-\sigma)}
$$

Thus the largest deviation of the temperature field from its free-stream value at the production layer is controlled by the value of the Prandtl number $\sigma$. In particular, if $\sigma<1$, then the deviation of the temperature field from its free-stream value is greater than the streamwise velocity deviation; this is again due to the relative thickness of the thermal and velocity boundary layers as discussed in $\S 4$.

It is also important to stress that, although the $\exp (-\sigma \xi)$ exponential in the basic temperature state $(4.5 a, b)$ dominates the decay of the basic state to its free-stream value, upon exiting the production layer towards the wall as $Y \rightarrow-\infty$, any growing temperature disturbances will be dominated by the $\exp (-2 Y)$ term in (5.1) and thus both exponentials need to be retained in the production-layer scalings.

Based on the discussion above, in the production layer we seek a solution of the Navier-Stokes equations in the form

$$
\left.\begin{array}{c}
(X, Y, z)=\left(x-c t, y-\ln R e+C_{0}, z\right) ; \quad c=1-\operatorname{Re}^{-1} c_{1}+\ldots, \\
\boldsymbol{u}=(1,0,0)+\operatorname{Re}^{-1} \overline{\boldsymbol{u}}(X, Y, z)+\ldots, \quad p=p_{\infty} / \rho_{\infty} u_{\infty}^{2}+\operatorname{Re}^{-2} \bar{p}(X, Y, z)+\ldots, \bar{\rho}^{-2}\left(\bar{\theta}_{2}, \bar{\rho}_{2}, \bar{\mu}_{2}\right)(X, Y, z) . \\
(\theta, \rho, \mu)=1+\operatorname{Re}^{-\sigma}\left(\bar{\theta}_{1}, \bar{\rho}_{1}, \bar{\mu}_{1}\right)(X, Y, z)+\operatorname{Re}^{-1},
\end{array}\right\}
$$

We substitute these scalings into the Navier-Stokes equations (3.2)-(3.5) and, at leading order, we obtain the production-layer problem

$$
\begin{gathered}
\mathcal{L} \overline{\boldsymbol{u}}=-\nabla \bar{p}+\nabla^{2} \overline{\boldsymbol{u}}, \text { at order } R e^{-1}, \\
\nabla \cdot \overline{\boldsymbol{u}}=0, \text { at order } \operatorname{Re}^{-1},
\end{gathered}
$$




\section{E.C. Johnstone and P. Hall}

$$
\begin{gathered}
\mathcal{L} \bar{\theta}_{1}=\sigma^{-1} \nabla^{2} \bar{\theta}_{1}, \text { at order } \operatorname{Re}^{-(\sigma+1)}, \\
\mathcal{L} \bar{\theta}_{2}=(\gamma-1) M_{\infty}^{2} \mathcal{L} \bar{p}+(\gamma-1) M_{\infty}^{2} \bar{\Phi}+\sigma^{-1} \nabla^{2} \bar{\theta}_{2}, \text { at order } \operatorname{Re}^{-3}, \\
\bar{\rho}_{1}+\bar{\theta}_{1}=0, \text { at order } R^{-\sigma}, \\
\bar{p}=R \theta_{\infty} u_{\infty}^{-2}\left(\bar{\rho}_{2}+\bar{\theta}_{2}\right), \text { at order } \operatorname{Re}^{-2}, \\
\bar{\mu}_{1}=\zeta \bar{\theta}_{1}, \text { at order } \operatorname{Re}^{-\sigma}, \\
\bar{\mu}_{2}=\zeta \bar{\theta}_{2}, \text { at order } \operatorname{Re}^{-2},
\end{gathered}
$$

where the operator $\mathcal{L}=\left(\left[\overline{\boldsymbol{u}}+c_{1} \hat{\boldsymbol{i}}\right] \cdot \nabla\right), \nabla=\left(\partial_{X}, \partial_{Y}, \partial_{z}\right)$ and the dissipation function $\bar{\Phi}$ is found by substituting the production-layer scalings into (3.6).

We see that the production-layer equations for the velocity field $\overline{\boldsymbol{u}}(5.4)-(5.5)$ are the same as the equations (2.2)-(2.3) for the incompressible production-layer problem in Deguchi \& Hall (2014), which describe a unit-Reynolds-number eigenvalue problem for the wave speed $c_{1}$. The only difference in the compressible problem is that the equations are solved at a slightly different value of $y$. Therefore, the solution to the incompressible eigenvalue problem, which was calculated in Deguchi \& Hall (2014), can now also be used for the compressible problem. The velocity field then drives the temperature field through the heat equations (5.6)-(5.7); (5.6), which is obtained at $O\left(R e^{-\sigma}\right)$, is dominant in the production layer, but we require the solution of the equation at $O\left(R e^{-2}\right)$ as the production layer is exited towards the wall.

The production-layer problem (5.4)-(5.11) is solved subject to boundary conditions specifying that the flow exiting the production layer on either side must match asymptotically onto the basic solution $(4.5 a, b)$,

$$
\begin{gathered}
\overline{\boldsymbol{u}} \rightarrow(0,-1,0), \quad \bar{\theta}_{1} \rightarrow \lambda \mathrm{e}^{-\sigma Y}, \quad \bar{\theta}_{2} \rightarrow-\lambda \mathrm{e}^{-2 Y} \quad \text { as } Y \rightarrow \infty, \\
\overline{\boldsymbol{u}} \rightarrow\left(-\mathrm{e}^{-Y},-1,0\right), \quad \bar{\theta}_{1} \rightarrow \lambda \mathrm{e}^{-\sigma Y}, \quad \bar{\theta}_{2} \rightarrow-\lambda \mathrm{e}^{-2 Y} \quad \text { as } Y \rightarrow-\infty,
\end{gathered}
$$

and periodicity conditions; defining $\alpha$ and $\beta$ as the streamwise and spanwise wavenumbers, respectively,

$$
\begin{aligned}
& \left(\overline{\boldsymbol{u}}, \bar{\theta}_{1,2}\right)(X, Y, z)=\left(\overline{\boldsymbol{u}}, \bar{\theta}_{1,2}\right)(X+2 \pi / \alpha, Y, z), \\
& \left(\overline{\boldsymbol{u}}, \bar{\theta}_{1,2}\right)(X, Y, z)=\left(\overline{\boldsymbol{u}}, \bar{\theta}_{1,2}\right)(X, Y, z+2 \pi / \beta) .
\end{aligned}
$$

Thus, boundary condition (5.13) allows for the streamwise velocity disturbance $\bar{u}$ to grow exponentially beneath the production layer. However, it also allows for the disturbances to the temperature field $\bar{\theta}_{1}, \bar{\theta}_{2}$ to grow exponentially, and at a faster rate than the streamwise velocity disturbance. Coming out of the production layer $\bar{\theta}_{2}$ is dominant, however, $\bar{\theta}_{1}$, which satisfies a homogeneous equation, must be retained as it is needed at the wall. All disturbances must be reduced to zero at the wall and therefore, as in the incompressible problem, the maximum value of the disturbances will occur in a layer between the wall and the production layer where the basic flow adjusts to accommodate the disturbance.

\section{The adjustment-layer problem}

Below the production layer, the flow returns to the unperturbed boundary-layer flow $(4.5 a, b)-(4.7 a, b)$ at leading order. However, the nonlinear production-layer interaction produces exponentially growing disturbances to the streamwise velocity and temperature fields that interact with the basic flow beneath the production layer. The flow between the 


\section{Free-stream coherent structures in compressible shear flows}

production layer and the wall adjusts to accommodate the disturbances; we thus refer to this region as the adjustment layer. The solution in the upper part of this layer is dominated by the solution exiting the production layer. Then as the wall is approached, the solution is described by the boundary-region equations.

\subsection{The solution exiting the production layer}

Firstly, above the production layer as $Y \rightarrow \infty$, the velocity must eventually return to its free-stream form $\overline{\boldsymbol{u}}=(0,-1,0)$. As in Deguchi \& Hall (2014), the decay of the streamwise velocity $u$ will be proportional to $\mathrm{e}^{-Y-C_{0}}$, however, the nonlinear interaction in the production layer gives a constant of proportionality which differs from unity. Thus the production-layer interaction can give at most an $O(1)$ effect on the amplitude of the streamwise velocity displacement. Since the temperature field in the production layer is entirely driven by the equations for the velocity (5.4)-(5.5), any temperature disturbances will also decay above the production layer as there is no interaction to sustain them.

We now consider $Y \rightarrow-\infty$. To analyse the flow beneath the production layer, we decompose the velocity disturbance $\overline{\boldsymbol{u}}$ into vortex and wave components. The wave is associated with the $X$-dependent components of the velocity field. The $X$-independent components of the velocity are split into a roll flow, which is associated with the components $\bar{v}$ and $\bar{w}$, and the streak, which is the downstream velocity component $\bar{u}$. The combination of the roll and streak constitutes a streamwise vortex. At leading order, the flow must satisfy the basic ASBL flow given by (5.13), and therefore we split the streak into a mean in $z$ and a $z$-dependent component (there is no mean in $z$ of the roll flow due to symmetry). In addition to the $z$-dependent components, we allow the $z$-independent term to grow exponentially in the adjustment layer as $Y \rightarrow-\infty$, but it must eventually at leading order reduce to $-\mathrm{e}^{-Y}$ in order to match onto the unperturbed basic flow at the wall.

We decompose the temperature disturbances $\bar{\theta}_{1}$ and $\bar{\theta}_{2}$ in the same way. Following the nomenclature outlined in Ricco \& Wu (2007), we refer to the $X$-independent component of the temperature disturbance as a 'thermal streak' and the corresponding streamwise velocity disturbance shall be termed a 'velocity streak'. Hence, in the adjustment layer, we seek a solution in the form

$$
\begin{gathered}
\overline{\boldsymbol{u}}=\left(\bar{u}_{S}(Y),-1,0\right)+\left(u_{S}(Y, z), v_{r}(Y, z), w_{r}(Y, z)\right)+\boldsymbol{u}_{w}(X, Y, z), \\
\bar{\theta}_{1,2}=\bar{\theta}_{s_{1,2}}(Y)+\theta_{s_{1,2}}(Y, z)+\theta_{w_{1,2}}(X, Y, z),
\end{gathered}
$$

where subscripts $s, r$ and $w$ refer to streak, roll and wave components respectively.

As in the incompressible ASBL study of Deguchi \& Hall (2014), outside of the production layer the roll flow decays as there is no longer any forcing from the Reynolds stresses associated with the wavefield to sustain it. The wave $\boldsymbol{u}_{w}$ also decays faster than the roll; this can be seen through a balance of advection-diffusion terms and is confirmed by the numerical results of Deguchi \& Hall (2014). Since the temperature field is driven entirely by the velocity field, the same is true of the corresponding temperature components $\theta_{w_{1,2}}$ and $\theta_{w_{1,2}}$. However, the velocity streak $\bar{u}_{s}+u_{s}$ can grow exponentially through interaction with the roll. The growth or decay of the velocity streak depends on the spanwise wavenumber $\beta$ through the periodicity conditions (5.15). The new feature for the compressible problem is that the interaction of the roll flow with the temperature field drives the growth of the thermal streak.

We substitute the decomposition of the disturbances (6.1)-(6.2) into the production-layer equations (5.4)-(5.11). After introducing the roll-flow streamfunction $\psi$ such that 


\section{E.C. Johnstone and P. Hall}

$\partial_{z} \psi=v_{r}$ and $\partial_{y} \psi=-w_{r}$, the resulting equations for the roll-velocity-streak flow are given by

$$
\begin{gathered}
\left(\frac{\partial^{2}}{\partial Y^{2}}+\frac{\partial}{\partial Y}+\frac{\partial^{2}}{\partial z^{2}}\right) u_{s}=\mathrm{e}^{-Y} v_{r} \\
\left(\frac{\partial^{2}}{\partial Y^{2}}+\frac{\partial}{\partial Y}+\frac{\partial^{2}}{\partial z^{2}}\right)\left(\frac{\partial^{2}}{\partial Y^{2}}+\frac{\partial^{2}}{\partial z^{2}}\right) \psi=0 \\
\left(\frac{\mathrm{d}}{\mathrm{d} Y}+\frac{\mathrm{d}^{2}}{\mathrm{~d} Y^{2}}\right) \bar{u}_{s}=\frac{\beta}{2 \pi} \frac{\mathrm{d}}{\mathrm{d} Y} \int_{z=0}^{2 \pi / \beta}\left(u_{s} v_{r}\right) \mathrm{d} z
\end{gathered}
$$

where the final equation for the mean velocity streak disturbance $\bar{u}_{s}$ has been found by taking the mean in $z$ of the production-layer $x$-momentum equation (5.4). It is important to note the $\partial_{Y}$ terms in the equations above which arise from the suction in the flow. It is these terms that allow the interaction of the mean part of the basic flow with the roll flow to produce growth.

The roll-velocity-streak equations (6.3)-(6.5) are solved together with the equations for the thermal streak,

$$
\begin{gathered}
\left(\frac{\partial^{2}}{\partial Y^{2}}+\frac{1}{\sigma} \frac{\partial}{\partial Y}+\frac{1}{\sigma} \frac{\partial^{2}}{\partial z^{2}}\right) \theta_{s_{1}}=-\sigma \lambda \mathrm{e}^{-\sigma Y} v_{r} \\
\left(\frac{\partial^{2}}{\partial Y^{2}}+\frac{1}{\sigma} \frac{\partial}{\partial Y}+\frac{1}{\sigma} \frac{\partial^{2}}{\partial z^{2}}\right) \theta_{s_{2}}=-2 \lambda v_{r} \mathrm{e}^{-2 Y}-2(\gamma-1) M_{\infty}^{2} \mathrm{e}^{-Y} \frac{\partial u_{s}}{\partial Y} \\
\left(\frac{\mathrm{d}}{\mathrm{d} Y}+\frac{1}{\sigma} \frac{\mathrm{d}^{2}}{\mathrm{~d} Y^{2}}\right) \bar{\theta}_{s_{1}}=\frac{\beta}{2 \pi} \frac{\mathrm{d}}{\mathrm{d} Y} \int_{z=0}^{2 \pi / \beta}\left(v_{r} \theta_{s_{1}}\right) \mathrm{d} z \\
\left(\frac{\mathrm{d}}{\mathrm{d} Y}+\frac{1}{\sigma} \frac{\mathrm{d}^{2}}{\mathrm{~d} Y^{2}}\right) \bar{\theta}_{v_{2}}=\frac{\beta}{2 \pi} \frac{\mathrm{d}}{\mathrm{d} Y} \int_{z=0}^{2 \pi / \beta}\left(v_{r} \theta_{s_{2}}-(\gamma-1) M_{\infty}^{2} \Phi_{v}\right) \mathrm{d} z
\end{gathered}
$$

where the dissipation function $\Phi_{v}$ associated with the vortex flow is

$$
\begin{aligned}
\Phi_{v}= & \frac{4}{3}\left(\frac{\partial v_{r}}{\partial Y}\right)^{2}+\frac{4}{3}\left(\frac{\partial w_{r}}{\partial z}\right)^{2}+\left(\frac{\mathrm{d} \bar{u}_{s}}{\mathrm{~d} Y}+\frac{\partial u_{s}}{\partial Y}\right)^{2}+\left(\frac{\partial u_{s}}{\partial z}\right)^{2}+\left(\frac{\partial v_{r}}{\partial z}\right)^{2} \\
& +2 \frac{\partial v_{r}}{\partial z} \frac{\partial w_{r}}{\partial Y}+\left(\frac{\partial w_{r}}{\partial Y}\right)^{2}-\frac{4}{3} \frac{\partial v_{r}}{\partial Y} \frac{\partial w_{r}}{\partial z} .
\end{aligned}
$$

These equations are solved by Fourier expansion in $z$. The numerical results of Deguchi $\&$ Hall (2014) show that the vortex wavelength is half that of the wave part of the flow, and therefore the wavelength of the vortex is $\pi / \beta$, which sets the wavenumbers of the Fourier expansion. Therefore, we seek a solution for $\psi$ in the form

$$
\psi=\sum_{n=0}^{\infty} a_{n} \cos (2 n \beta z)+\sum_{n=1}^{\infty} b_{n} \sin (2 n \beta z) .
$$

The roll-velocity-streak equations (6.3)-(6.5) are the same as those for the incompressible equation in Deguchi \& Hall (2014), with $Y=y-\ln R e+C_{0}$ where $C_{0}=0$ (corresponding to $M_{\infty}=0$ ) in the incompressible problem. Thus, the solution of (6.3)-(6.5) is the same as that for the incompressible problem; the incompressible solution with $C_{0}=0$ is given in (2.4)-(2.6). Thus upon exiting the production layer in the 
compressible problem, the leading order solution of (6.3)-(6.5) in original boundary-layer coordinates $(x, y, z)$ and associated flow quantities $(u, v, w)$ is

$$
\begin{gathered}
u \rightarrow 1-\exp \left(-\left(y+C_{0}\right)\right)+\frac{d_{0}}{R e}+\frac{J_{1}}{R e^{\omega_{1}}} \exp \left(\left(\omega_{1}-1\right)\left(y+C_{0}\right)\right) \cos (2 \beta z) \\
+\frac{J_{1} K_{1}}{R e^{2 \omega_{1}} 4 \omega_{1}} \exp \left(\left(2 \omega_{1}-1\right)\left(y+C_{0}\right)\right)+\cdots, \\
v \rightarrow-\frac{1}{R e}+\frac{K_{1}}{R e^{\omega_{1}+1}} \exp \left(\omega_{1}\left(y+C_{0}\right)\right) \cos (2 \beta z)+\cdots, \\
w \rightarrow-\frac{K_{1} \omega_{1}}{2 \beta \operatorname{Re}^{\omega_{1}+1}} \exp \left(\omega_{1}\left(y+C_{0}\right)\right) \sin (2 \beta z)+\cdots,
\end{gathered}
$$

where

$$
J_{n}=\frac{K_{n}}{\left(\omega_{n}-1\right)^{2}+\left(\omega_{n}-1\right)-4 n^{2} \beta^{2}}, \quad \omega_{n}=\frac{-1+\sqrt{1+16 n^{2} \beta^{2}}}{2} \geqslant 0,
$$

for $n \geqslant 1$. The terms represented by '. '. represent more slowly growing harmonics in $z$, with constants $J_{n}, K_{n}$ and $\omega_{n}$ for $n>1$. The constants $d_{0}$ and $K_{1}$ are found as part of the nonlinear eigenvalue production-layer problem; $K_{1}$ was reported for a range of $\beta$ in Deguchi \& Hall (2014). Thus we only give the full streamwise velocity solution up to a constant $d_{0} / R e$, but this constant does not affect the streaks. As required, the flow returns to its unperturbed basic state at leading order, with exponentially growing disturbances that can become larger than the velocities involved in the nonlinear interaction in the production layer where the disturbances originated.

The solutions for $u_{s}, v_{r}$ and $\bar{u}_{s}$ are then used as forcing for the equations (6.6)-(6.9) for the thermal streak. In the original boundary-layer variables, $\theta=1+R e^{-\sigma} \bar{\theta}_{1}+R e^{-2} \bar{\theta}_{2}$, we find that upon exiting the production layer,

$$
\begin{aligned}
\theta & \rightarrow 1+\lambda \exp \left(-\sigma\left(y+C_{0}\right)\right)-\lambda \exp \left(-2\left(y+C_{0}\right)\right)+\frac{d_{1}}{R e^{\sigma}}+\frac{d_{2}}{R e^{2}} \\
& +\frac{1}{R e^{\omega_{1}}}\left(L_{1} \exp \left(\left(\omega_{1}-\sigma\right)\left(y+C_{0}\right)\right)+Q_{1} \exp \left(\left(\omega_{1}-2\right)\left(y+C_{0}\right)\right)\right) \cos (2 \beta z) \\
& +\frac{1}{R e^{2 \omega_{1}}}\left(\frac{L_{1} K_{1} \sigma}{4 \omega_{1}} \exp \left(\left(2 \omega_{1}-\sigma\right)\left(y+C_{0}\right)\right)+R_{1} \exp \left(\left(2 \omega_{1}-2\right)\left(y+C_{0}\right)\right)\right)+\cdots,
\end{aligned}
$$

where again the terms represented by '...' denote more slowly growing harmonics in $z$, with constants $K_{n}, J_{n}, L_{n}, Q_{n}, R_{n}$ and $\omega_{n}$ for $n>1$ and where

$$
\begin{gathered}
L_{n}=\frac{-K_{n} \lambda \sigma}{\left(\omega_{n}-\sigma\right)+\sigma^{-1}\left(\omega_{n}-\sigma\right)^{2}-\sigma^{-1} 4 n^{2} \beta^{2}}, \\
Q_{n}=\frac{-2 \lambda K_{n}-2(\gamma-1) M_{\infty}^{2} J_{n}\left(\omega_{n}-1\right)}{\left(\omega_{n}-2\right)+\sigma^{-1}\left(\omega_{n}-2\right)^{2}-\sigma^{-1} 4 n^{2} \beta^{2}}, \\
R_{n}=-2 \frac{\sigma\left(\frac{1}{4} M_{\infty}^{2} J_{n}^{2}(\gamma-1) \omega_{n}^{3}-\frac{1}{2} M_{\infty}^{2} J_{n}^{2}(\gamma-1) \omega_{n}^{2}-\frac{1}{4} J_{n} M_{\infty}^{2} K_{n}(\gamma-1)\right)}{\omega_{n}\left(\sigma+2 \omega_{n}-2\right)} \\
\left.-2 \frac{\sigma\left(M_{\infty}^{2}\left(\beta^{2} n^{2}+\frac{1}{4}\right)(\gamma-1) J_{n}^{2}+\frac{1}{2} J_{n} M_{\infty}^{2} K_{n}(\gamma-1)-\frac{1}{4} Q_{n} K_{n}\right)}{\sigma+2 \omega_{n}-2} .176 .17 c\right)
\end{gathered}
$$




$\begin{array}{ccccc} & (a) \beta<\frac{\sqrt{3}}{4} & (b) \frac{\sqrt{3}}{4}<\beta<\frac{1}{\sqrt{2}} & \text { (c) } \frac{1}{\sqrt{2}}<\beta<\sqrt{\frac{3}{2}} & (d) \beta>\sqrt{\frac{3}{2}} \\ u_{s} & \mathrm{G} & \mathrm{G} & \mathrm{D} & \mathrm{D} \\ \bar{u}_{s} & \mathrm{G} & \mathrm{D} & \mathrm{D} & \mathrm{D} \\ \theta_{s} & \mathrm{G} & \mathrm{G} & \mathrm{G} & \mathrm{D} \\ \bar{\theta}_{v} & \mathrm{G} & \mathrm{G} & \mathrm{D} & \mathrm{D}\end{array}$

Table 1. The growth and decay of the disturbances for different values of the spanwise wavenumber $\beta$. Growth is represented by ' $\mathrm{G}$ ' and decay by ' $\mathrm{D}$ '. The growth and decay is shown for the both the mean in $z$ and the $z$-dependent parts of the flow.

The constants $d_{1}$ and $d_{2}$ are constants of integration; again, we only find the solution for the temperature field up to a constant, but this constant does not affect the growth of the thermal streaks beneath the production layer.

The asymptotic solution (6.12)-(6.13) for $u$ and $v$ beneath the production layer shows that the roll flow always decays as the wall layer is approached, whereas the mean part of the velocity streak flow (6.12) can grow beneath the production layer if $2 \omega_{1}<1$, corresponding to values of $\beta<\sqrt{3} / 4$. The $z$-dependent part of the velocity streak can grow if $\omega_{1}<1$, corresponding to values of $\beta<1 / \sqrt{2}$, and therefore these latter modes are the fastest growing. If $\beta>1 / \sqrt{2}$, then the velocity streak disturbance decays exponentially, and the nonlinear interaction in the production layer simply produces an $O\left(R e^{-1}\right)$ correction to the flow.

Meanwhile, the asymptotic solution for the temperature (6.16) beneath the production layer shows that the thermal streaks can grow if $\omega_{1}<\sigma$ or if $\omega_{1}<2$. For the range of values of Prandtl number $\sigma<2$ considered, the modes proportional to $\exp \left(\left(\omega_{1}-2\right)\right.$ $\left.\left(y+C_{0}\right)\right)$ will dominate the growth, and therefore the nonlinear interaction in the production layer will always produce growing temperature disturbances for $\beta<\sqrt{3} / \sqrt{2}$.

The structure of the solution with varying $\beta$ is summarised in table 1 . The asymptotic results suggest that there exists a case where the thermal streaks can grow while the velocity streak decays. However, solutions of the production-layer problem (5.4)-(5.5) have not been found for values of $\beta \gtrsim 0.47$ (Deguchi \& Hall 2014, 2015), and therefore cases $(c)$ and $(d)$ are possibly not relevant.

We see that, in all cases, a nonlinear interaction in the production layer of size $O\left(R e^{-1}\right)$, which drives $O\left(R e^{-2}\right), O\left(R e^{-\sigma}\right)$ temperature perturbations, can induce much larger changes to the velocity and temperature fields of $O\left(R e^{-\omega_{1}}\right)$ in the main part of the boundary layer. We now consider the solution as it approaches the wall layer, where all disturbances are eventually reduced to zero to satisfy the wall boundary conditions.

\subsection{Boundary-layer analysis}

The solutions exiting the production layer, (6.12)-(6.14) and (6.16), do not satisfy the wall boundary conditions. We now find the solution for the induced flow which is valid all the way down to the wall. This solution should also match onto the solution exiting the production layer given by (6.12)-(6.14) and (6.16). An examination of this solution shows that in the boundary layer, disturbances can grow exponentially. The $z$-dependent part of the disturbance grows faster than the z-independent part; therefore, to match onto the solution exiting the production layer, the boundary-region solution will have $z$-dependence 


\section{Free-stream coherent structures in compressible shear flows}

in the form of $\cos (2 \beta z)$. However, the solution must also satisfy conditions (4.2a), and therefore any disturbances must ultimately be reduced to zero at the wall.

The solution in the boundary region is described in terms of the Dorodnitsyn-Howarth variable $\xi$; since disturbances are always small compared with the basic flow, the definition of the variable in (4.3) is valid throughout the flow, and in particular, $\partial_{y}=\hat{\rho}(\xi) \partial \xi$. The inversion of the Dorodnitsyn-Howarth transformation for $y(\xi)$ is given in (4.8), however, unlike near the wall and in the production layer, we cannot generally find an explicit relationship for $\xi(y)$ as it cannot be assumed that the exponential terms involving $\xi$ are smaller than the linear terms. Therefore, to find the solution for the physical variable $y$, we first solve the boundary-region equations in terms of $\xi$, and then use the monotonic relationship $y(\xi)$ in (4.8) to plot the solutions for each corresponding value of $y$.

Based on this discussion, in the boundary region we seek a solution in terms of the fundamental harmonics of the solution exiting the production layer (6.12)-(6.14), (6.16) in the form

$$
\begin{gathered}
u=\hat{u}(\xi)+\operatorname{Re}^{-\omega_{1}} \tilde{u}(\xi) \cos (2 \beta z), \\
v(\xi)=\operatorname{Re}^{-1} \hat{v}(\xi)+\operatorname{Re}^{-\left(1+\omega_{1}\right)} \tilde{v}(\xi) \cos (2 \beta z), \\
w=\operatorname{Re}^{-\left(1+\omega_{1}\right)} \tilde{w}(\xi) \sin (2 \beta z), \quad p=\hat{p}(\xi)+\operatorname{Re}^{-\left(2+\omega_{1}\right)} \tilde{p}(\xi) \cos (2 \beta z), \\
(\theta, \rho, \mu)=(\hat{\theta}(\xi), \hat{\rho}(\xi), \hat{\mu}(\xi))+\operatorname{Re}^{-\omega_{1}}(\tilde{\theta}(\xi), \tilde{\rho}(\xi), \tilde{\mu}(\xi)) \cos (2 \beta z),
\end{gathered}
$$

where the basic solution (hat quantities) is given by the solution of $(4.4 a, b)$. We use the same velocity streak and thermal streak terminology to refer to the disturbances to the streamwise velocity and temperature fields respectively, and again the roll flow is associated with the disturbances to the $(v, w)$ components of the velocity.

We substitute this expansion into the Navier-Stokes equations (3.2)-(3.5), which leads to a set of ordinary differential equations in $\xi$ for the leading-order disturbance amplitudes (tilde quantities). Following Hall (1983), we eliminate the pressure $\tilde{p}$ and the spanwise disturbance velocity $\tilde{w}$; then we also eliminate the viscosity $\tilde{\mu}$ and the density $\tilde{\rho}$ using the equation of state (3.5) and the linearised power-law viscosity law (3.7). We are then left with three coupled differential equations for $\tilde{u}$ (from the $x$-momentum equation), $\tilde{v}$ (from the $y$-momentum equation) and $\tilde{\theta}$ (from the temperature equation)

$$
\begin{gathered}
A_{1} \tilde{u}+A_{2} \tilde{u}^{\prime}+A_{3} \tilde{u}^{\prime \prime}=A_{4} \tilde{v}+A_{5} \tilde{\theta}+A_{6} \tilde{\theta}^{\prime}, \\
B_{1} \tilde{v}+B_{2} \tilde{v}^{\prime}+B_{3} \tilde{v}^{\prime \prime}+B_{4} \tilde{v}^{(3)}+B_{5} \tilde{v}^{(4)}=B_{6} \tilde{\theta}+B_{7} \tilde{\theta}^{\prime}+B_{8} \tilde{\theta}^{\prime \prime}+B_{9} \tilde{\theta}^{(3)}+B_{10} \tilde{\theta}^{(4)}, \\
C_{1} \tilde{\theta}+C_{2} \tilde{\theta}^{\prime}+C_{3} \tilde{\theta}^{\prime \prime}=C_{4} \tilde{u}^{\prime}+C_{5} \tilde{v} .
\end{gathered}
$$

Here, the superscripts represent derivatives in the usual way. The coefficients $A_{k}, B_{k}$ and $C_{k}$ depend on the basic solution and are too long to write here; details are available from the authors on request. These coupled equations are solved subject to zero-disturbance and no-slip boundary conditions at the wall, and matching to the solution exiting the production layer (6.12), (6.13), (6.16) at $\xi=\xi_{P L}=\ln R e$, so that

$$
\begin{gathered}
\tilde{u}(0)=0, \quad \tilde{u}\left(\xi_{P L}\right)=J_{1} \exp \left(\left(\omega_{1}-1\right) \xi_{P L}\right), \\
\tilde{v}(0)=\tilde{v}^{\prime}(0)=0, \quad \tilde{v}\left(\xi_{P L}\right)=K_{1} \exp \left(\omega_{1} \xi_{P L}\right), \quad \tilde{v}^{\prime}\left(\xi_{P L}\right)=K_{1} \omega_{1} \exp \left(\omega_{1} \xi_{P L}\right), \\
\tilde{\theta}(0)=0, \quad \tilde{\theta}\left(\xi_{P L}\right)=\left(L_{1} \exp \left(\left(\omega_{1}-\sigma\right) \xi_{P L}\right)+Q_{1} \exp \left(\left(\omega_{1}-2\right) \xi_{P L}\right)\right) .
\end{gathered}
$$




$\begin{array}{lcccc}\text { Altitude }(\mathrm{m}) & \theta_{\infty}(\mathrm{K}) & a_{\infty}\left(\mathrm{m} \mathrm{s}^{-1}\right) & u_{\infty}\left(\mathrm{m} \mathrm{s}^{-1}\right) & v_{\infty}\left(\mathrm{m} \mathrm{s}^{-1}\right) \\ 0 & 288.2 & 340 & 34-679 & 2.4 \times 10^{-4}-8.5 \times 10^{-3} \\ 5000 & 255.7 & 320 & 32-640 & 2.3 \times 10^{-4}-8.0 \times 10^{-3} \\ 11000 & 216.8 & 295 & 29-589 & 2.1 \times 10^{-4}-7.4 \times 10^{-3}\end{array}$

Table 2 . The range of dimensional values of free-stream velocity $u_{\infty}$ and suction velocity $v_{\infty}$ at free-stream temperature $\theta_{\infty}$ for the range of Mach numbers $0.1 \leqslant M_{\infty} \leqslant 2$ and Reynolds numbers $80000 \leqslant R e \leqslant$ 140000 .

The reduced boundary-region equations are discretised on a grid with $N$ interior points and we use second-order accurate centred finite differences to approximate the derivatives with step size $\Delta \xi$; see Appendix A for details. We then solve the resulting matrix equation for $\tilde{u}, \tilde{v}$ and $\tilde{\theta}$.

\section{Results}

We solve the matrix system for $\tilde{u}, \tilde{v}$ and $\tilde{\theta}$ on a grid containing $N=2000$ points. To compute the boundary conditions (6.12), (6.13) and (6.16), we require the value of $K_{1}=$ $K_{1}(\alpha, \beta)$ which is determined as part of the numerical solution of the production-layer nonlinear eigenvalue problem (5.4)-(5.5) for the wave speed $c_{1}$. For wavenumber values $(\alpha, \beta)=(0.2,0.4)$, which by table 1 is in the regime where both the velocity and thermal streaks are expected to grow, Deguchi \& Hall (2014) find $K_{1}=16.9$; we use these parameter values in our computations.

We explore the behaviour of the velocity and thermal streaks as the Reynolds number $R e$, Mach number $M_{\infty}$ and Prandtl number $\sigma$ vary. The Reynolds number and Mach number are defined using the dimensional quantities $u_{\infty}$ (the streamwise velocity), $v_{\infty}$ (the suction velocity) and $\theta_{\infty}$ (the free-stream temperature). Using the International Standard Atmosphere (International Organization for Standardization 1975) value for temperature at a fixed altitude, we describe in table 2 the range of free-stream velocities $u_{\infty}$ and suction velocities $v_{\infty}$ required to obtain Reynolds numbers in the range 80 000-140 000 and Mach numbers in the subsonic to moderate supersonic range, $0.1 \leqslant M_{\infty} \leqslant 2$.

Next, to examine the development of the flow disturbances beneath the production layer, we define the amplitudes of the leading-order velocity streak, roll and thermal streak solutions exiting the production layer (6.12)-(6.14), (6.16) and the numerical solution in the boundary region

$$
\begin{aligned}
& A_{u_{s}}=\operatorname{Re}^{-1} \sqrt{\frac{\beta}{2 \pi} \int_{0}^{2 \pi / \beta} u_{s}^{2} \mathrm{~d} z}, \quad A_{\tilde{u}}=R e^{-\omega_{1}} \sqrt{\frac{\tilde{u}^{2}}{2}}, \\
& A_{v_{r}, w_{r}}=\operatorname{Re}^{-1} \sqrt{\frac{\beta}{2 \pi} \int_{0}^{2 \pi / \beta}\left(v_{r}^{2}+w_{r}^{2}\right) \mathrm{d} z}, \quad A_{\tilde{v}, \tilde{w}}=\operatorname{Re}^{-\left(\omega_{1}+1\right)} \sqrt{\frac{\tilde{v}^{2}+\tilde{w}^{2}}{2}}, \\
& A_{\theta_{s}}=\sqrt{\frac{\beta}{2 \pi} \int_{0}^{2 \pi / \beta}\left(\operatorname{Re}^{-\sigma} \theta_{s_{1}}+R e^{-2} \theta_{s_{2}}\right)^{2} \mathrm{~d} z}, \quad A_{\tilde{\theta}}=R e^{-\omega_{1}} \sqrt{\frac{\tilde{\theta}^{2}}{2}} .
\end{aligned}
$$

We first consider the validity of the upper adjustment-layer solution (6.12)-(6.14), (6.16). In figure 1 we plot the amplitudes of the velocity streaks, roll flow and thermal streaks for both the asymptotic solution (6.12)-(6.14), (6.16) and the numerical solution of the 

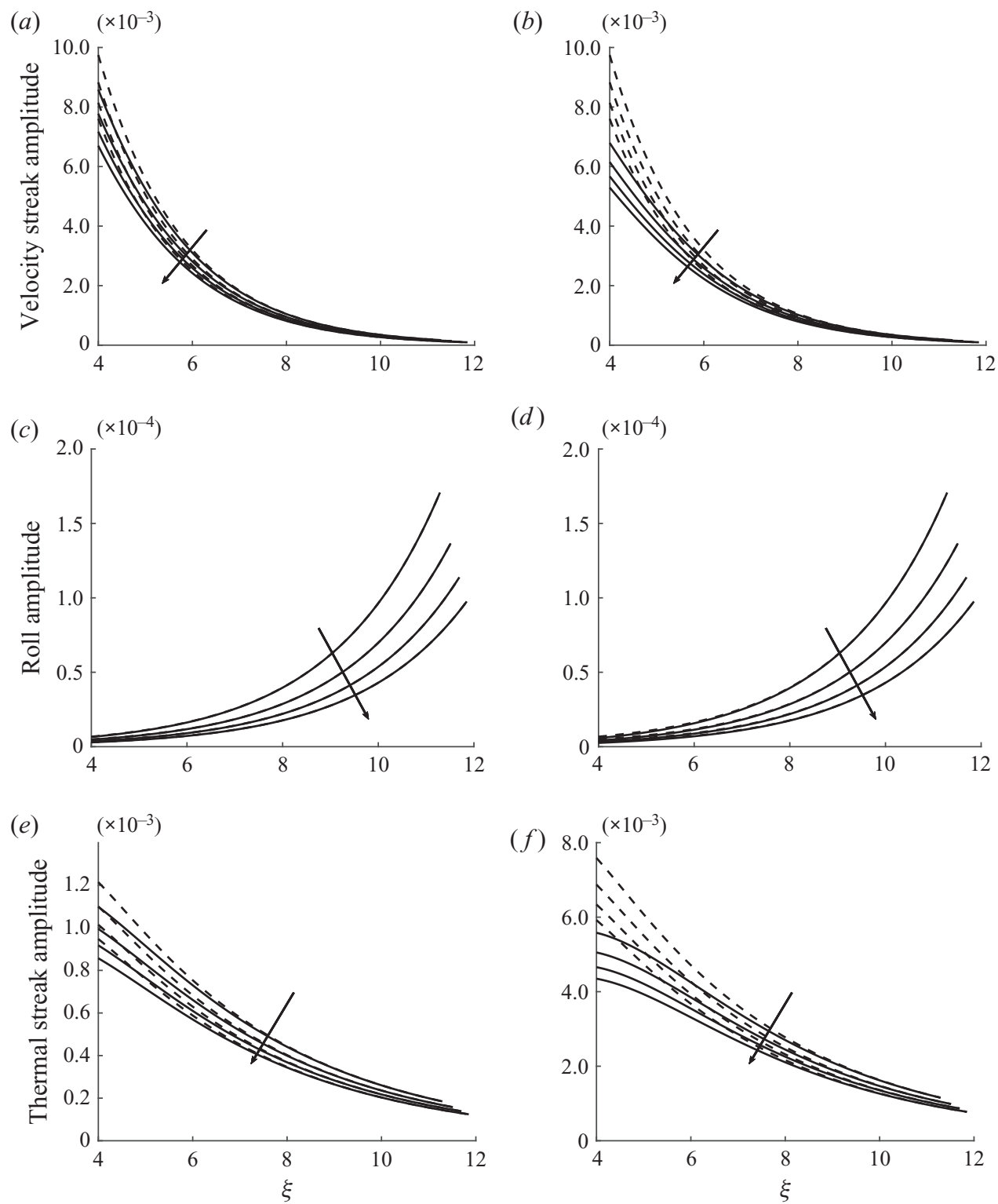

Figure 1. The amplitudes of the velocity streak $(a, b)$, roll flow $(c, d)$ and thermal streak $(e, f)$. The solid black lines denote the amplitudes of the boundary-region solution $\left(A_{\tilde{u}}, A_{\tilde{v}}, \tilde{w}, A_{\tilde{\theta}}\right)$ whilst the dashed lines denote the amplitudes of the asymptotic production-layer solution $\left(A_{u_{s}}, A_{v_{r}, w_{r}}, A_{\theta_{s}}\right)$ as functions of the Dorodnitsyn-Howarth variable $\xi$. The four lines denote the amplitudes calculated for Reynolds numbers $R e=80000,1000000,120000$ and 140000 , corresponding to production-layer locations $\xi_{P L}=11.29,11.51$, 11.70 and 11.85 respectively. The black arrow denotes the direction of increasing Reynolds number. The amplitudes in $(a, c, e)$ were calculated using a subsonic free-stream Mach number $M_{\infty}=0.8$ whilst $(b, d, f)$ are the amplitudes for the moderate supersonic regime with $M_{\infty}=2$. The Prandtl number is $\sigma=0.71$ and we have used $\zeta=0.76$ in the power-law viscosity law (3.7) to calculate the basic flow.

boundary-region equations (6.19)-(6.21) in terms of the Dorodnitsyn-Howarth variable $\xi$, so that the production layer is located at $\xi_{P L}=\ln R e$. For both the subsonic $(a, c, e)$ and moderate supersonic $(b, d, f)$ regimes, the asymptotic solution describing the roll flow is valid all the way to the wall, whereas the solution for the velocity and thermal streaks 


\section{E.C. Johnstone and P. Hall}
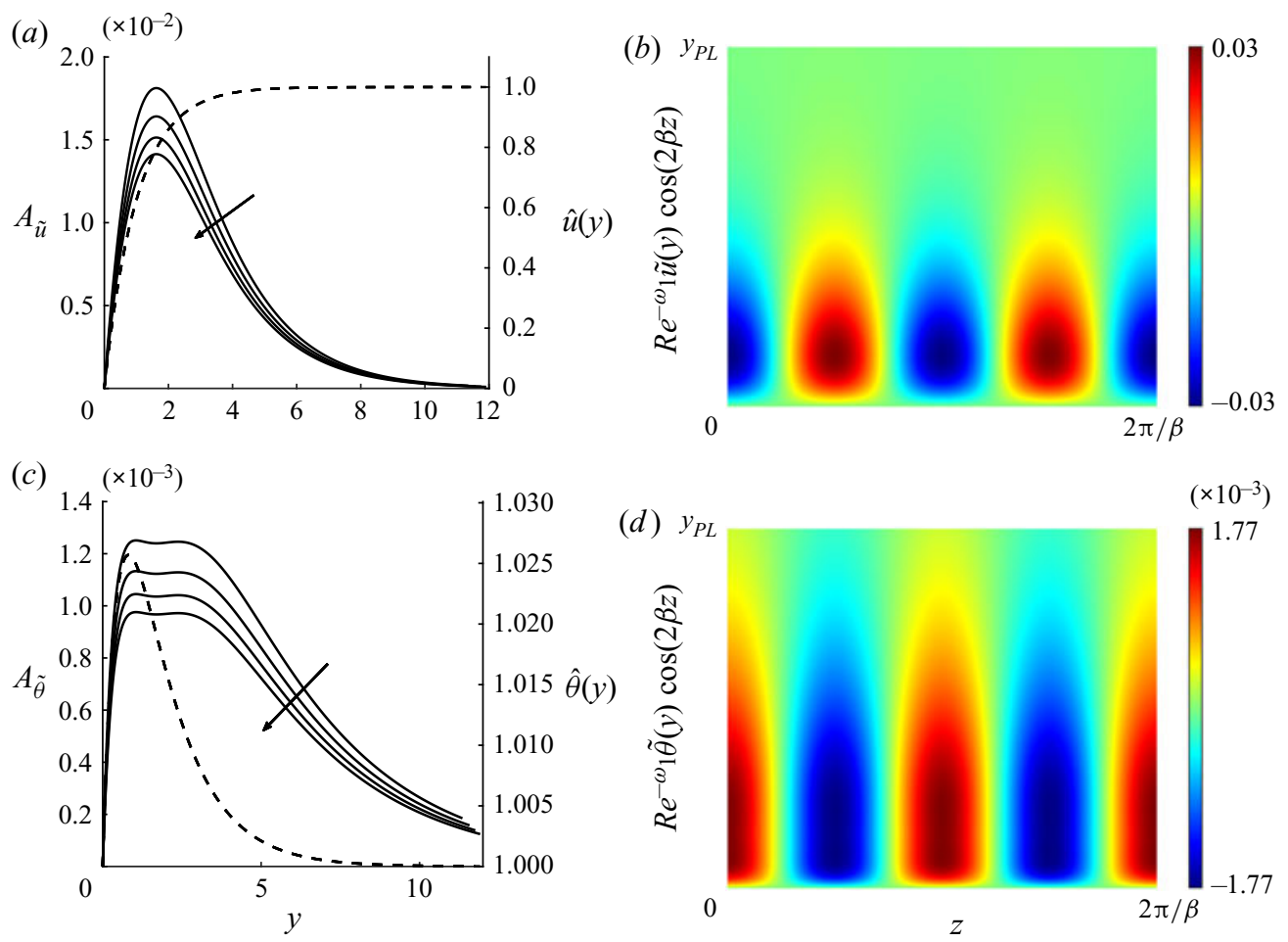

Figure 2. The numerical solution of boundary-region equations (6.19)-(6.21) at subsonic Mach number $M_{\infty}=0.8$. The Prandtl number is $\sigma=0.71$ and we have used $\zeta=0.76$ in the power-law viscosity law (3.7) to calculate the basic flow. The amplitudes of the velocity streak $A_{\tilde{u}}(a)$ and the thermal streak $A_{\tilde{\theta}}(c)$ (left axis) are denoted by solid black lines together with the basic flow profile $\hat{u}(y)(a)$ and $\hat{\theta}(y)$ (c) (right axis), which are denoted by dashed lines. The four solid lines denote the amplitudes calculated for Reynolds numbers $R e=80000,1000000,120000$ and 140000 , corresponding to production-layer locations $y_{P L}=11.35,11.58$, 11.76 and 11.91 respectively. The black arrow denotes the direction of increasing Reynolds number. The velocity $(b)$ and thermal $(d)$ streaks are shown over two vortex wavelengths at a Reynolds number $R e=80000$, with $y_{P L}=11.35$.

breaks down as the wall is approached; the location of this breakdown indicates the thickness of the upper adjustment layer. This breakdown occurs further from the wall in the moderate supersonic regime, indicating a thinner upper adjustment layer. We also note that the amplitude of the thermal streaks for subsonic free-stream Mach numbers is approximately one order of magnitude smaller than that of velocity streaks, whereas in the moderate supersonic regime the amplitudes are comparable.

As the wall is approached, the numerical solution of the boundary-region equations describes the flow induced by the disturbances from the production layer. In figure 2 we show the development of the amplitudes of the velocity and thermal streaks as the walls is approached, for subsonic free-stream Mach number $M_{\infty}=0.8$, as a function of the physical variable $y$ which is related to the Dorodnitsyn-Howarth variable by (4.8). As in the incompressible case, the velocity streak grows throughout the boundary region before taking its maximum in the near-wall boundary layer. In the compressible problem, the nonlinear interaction in the production layer also produces a thermal streak which similarly grows throughout the boundary layer; the rate growth of the thermal streak is higher than that of the velocity streak so that the effect of the thermal streak is felt both further from the wall and more uniformly across the flow compared with the 

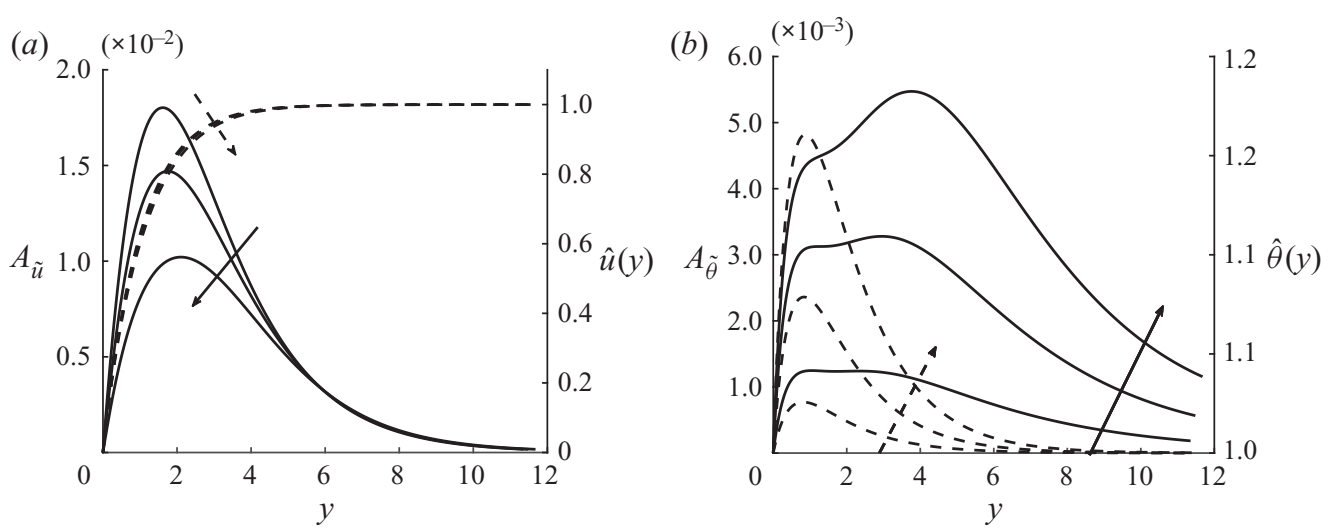

Figure 3. The amplitude of the velocity streaks $(a)$ and thermal streaks $(b)$ (solid lines, left axis), together with the basic flows $\hat{u}(y)$ and $\hat{\theta}(y)$ (dashed lines, right axis) for free-stream Mach numbers $M_{\infty}=0.8,1.4$ and 2 . The arrow indicates the direction of increasing Mach number. The Prandtl number is $\sigma=0.71$ and we have used $\zeta=0.76$ in the power-law viscosity law (3.7) to calculate the basic flow.

velocity streak. In figure $2(b, d)$ we show the velocity and thermal streaks over two vortex wavelengths. Note that the velocity and thermal streaks shown in figure $2(b, d)$ are in phase, but the functions of $y$ multiplying $\cos (2 \beta z)$ have opposite sign.

The variation of the amplitude of the velocity and thermal streaks for varying Mach number is shown in figure 3, for a Reynolds number of $R e=80000$. The amplitude of the thermal streak is enhanced as the free-stream Mach number is increased whilst the amplitude of the velocity streaks decreases; as noted above, for moderate supersonic $M_{\infty}$ the amplitudes are of comparable magnitude. This is consistent with the idea of compressibility effects becoming more important as the free-stream Mach number is increased (Morkovin 1962), but also suggests that the amplitude of the velocity streaks could be become larger than that of the thermal streaks in more compressible regimes. The location of the maximum amplitude of the thermal streak occurs further from the wall as $M_{\infty}$ is increased, with the structure of the amplitude solution changing from two local maxima to one more pronounced peak. Thus unlike the incompressible case, the growth of the thermal streak is not uniform in $y$.

Meanwhile, the effect of Prandtl number on the streak amplitude is shown in figure 4 for both the subsonic and moderate supersonic regimes. Increasing the Prandtl number from 0.7 to 1.3 leads to velocity streaks with smaller maximum amplitude where the maximum occurs further from the wall; these effects are more pronounced in the moderate supersonic regime than the subsonic regime. Meanwhile, for the thermal streaks, the effect of increasing the Prandtl number is to decrease the amplitude of the streak exiting the production layer, inhibit the growth of the streak further from the wall, but increase the eventual rate of growth. In the subsonic case the streaks eventually have a larger maximum amplitude; this is not the case in the moderate supersonic regime.

\section{Discussion}

Our results show the existence of free-stream coherent structures in the compressible ASBL at $O(1)$ Mach number. The solutions take the form of a roll-wave-streak interaction at the edge of the boundary layer, in a production layer whose location is dependent on both the Prandtl number and the Mach number. The interaction produces both a streaky disturbance and a temperature disturbance. These grow exponentially out of the production 

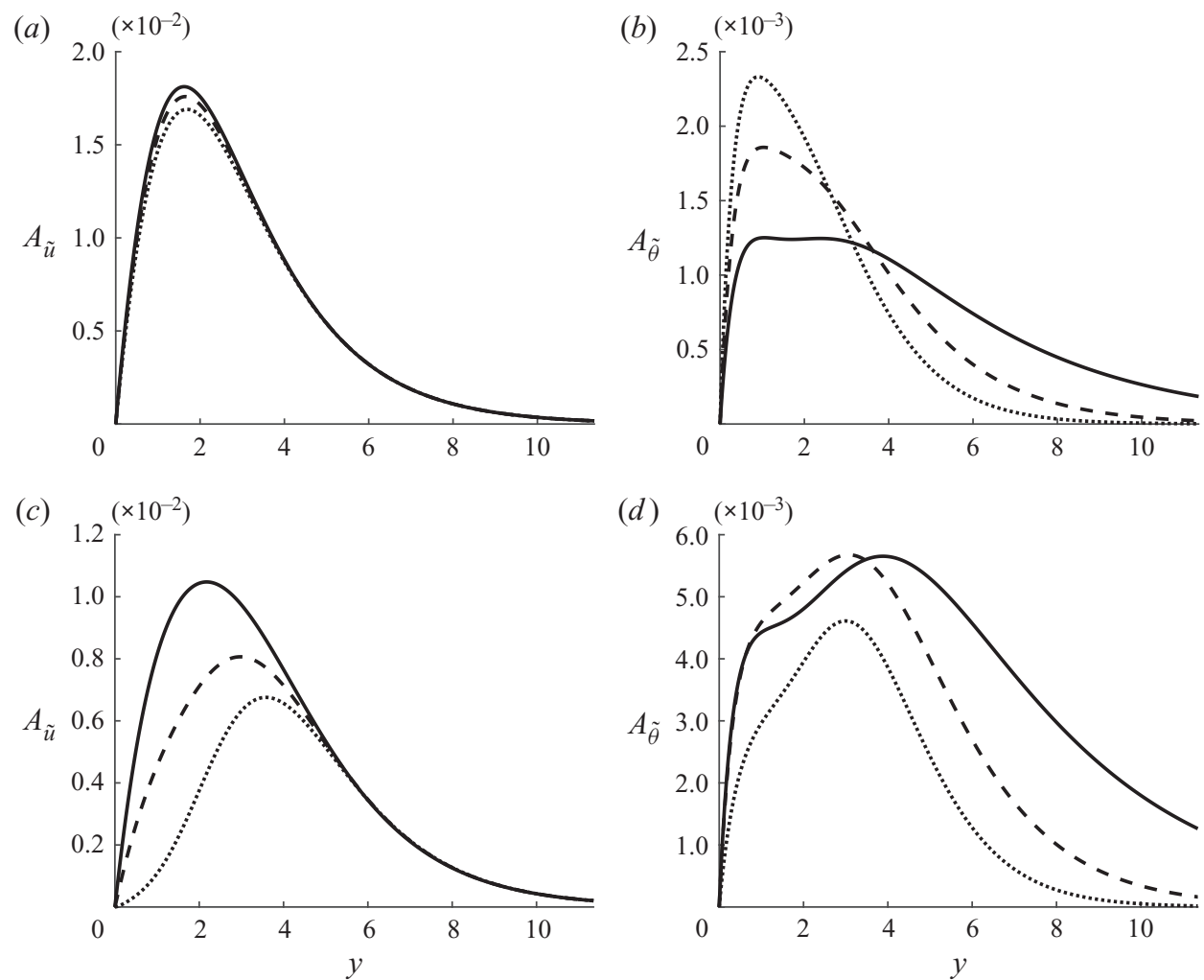

Figure 4. The amplitude of the velocity streaks $(a, c)$ and thermal streaks $(b, d)$ for Prandtl number $\sigma=0.71$ (solid black line), 1 (dashed line) and 1.3 (dotted line). The amplitudes in $(a, c)$ are for a subsonic free-stream Mach number $M_{\infty}=0.8$ whilst $(b, d)$ are for the moderate supersonic regime with $M_{\infty}=2$. The Reynolds number is $R e=80000$ and we have used $\zeta=0.76$ in the power-law viscosity law (3.7) to calculate the basic flow.

layer, with the rate of growth being controlled by the spanwise wavenumber and, for the temperature disturbance, the Prandtl number. Above the layer, the disturbances decay rapidly to zero. For the compressible case considered here, the main difference from the incompressible case is the development of a spanwise varying temperature field beneath the production layer. The amplitude of the induced temperature field disturbances depends on both the Prandtl number and the free-stream Mach number, with the amplitude of the velocity and thermal streaks being comparable in the moderate supersonic regime. We might anticipate that in practice the induced temperature and streak fields could be big enough to lead to secondary instabilities. In the incompressible case we know from Dempsey, Hall \& Deguchi (2017) that the streak generated by the free-stream coherent structure acts as a receptivity mechanism in curved flows, so for curved compressible flows, such as those over turbine blades, we anticipate that the structures described here might trigger transition through the Görtler vortex mechanism.

Our results show that the fundamental mechanism described by Deguchi \& Hall (2014) for incompressible flows is also operational in compressible flows. In particular, this suggests the mechanism will occur in compressible jets and therefore might have important consequences for sound production in compressible jet flows. Extension of the work of Deguchi \& Hall (2015) on swept wing flows is also possible. 


\section{Free-stream coherent structures in compressible shear flows}

Our analysis has assumed that the viscosity can be described by the power-law viscosity law, that the effect of shocks in the moderate supersonic regime is negligible with a sufficiently thin plate and that the gas in question is an ideal gas. Extension of the work to account for a more realistic viscosity model, for example Sutherland's law (Sutherland 1893), is straightforward but we believe that for the Mach numbers considered here that is not necessary. At hypersonic speeds beyond the regime covered here both real gas effects and more realistic viscosity models must be used and an intriguing question is the relationship between the production-layer problem and the temperature adjustment layer for the basic state at hypersonic speeds. Certainly, we know from for example Cowley \& Hall (1990), Blackaby, Cowley \& Hall (1993) and Fu, Hall \& Blackaby (1993) that real gas effects, realistic viscosity models and indeed shocks present in the flow can significantly alter streamwise vortex or travelling-wave instabilities, so it is to be expected that the free-stream coherent structure mechanism at hypersonic speeds will be significantly different from that in the moderate supersonic case.

It is not yet known whether the class of exact coherent structures described by Hall \& Sherwin (2010) can be extended to compressible flows. However, the fundamental asymptotic analysis supporting the structure is the vortex-wave interaction theory of Hall \& Smith (1991) which in fact was given in the context of compressible flows so it would appear likely that it is operational in compressible flows. Moreover, the inviscid stability equation for many boundary-layer compressible flows has unstable solutions when the incompressible counterpart has none (Mack 1975, 1984) so it may well be that vortex-wave interactions in compressible flows may have a richer structure than their incompressible counterparts. Taken together with the extension of the free-stream coherent structure mechanism to compressible flows, it suggests that compressible flows might well have a significant family of possible exact coherent states.

Acknowledgements. The authors would like to thank the referees for their helpful and constructive comments.

Declaration of interests. The authors report no conflict of interest.

\section{Author ORCIDs.}

(D) Eleanor C. Johnstone https://orcid.org/0000-0002-2166-5859;

D Philip Hall https://orcid.org/0000-0001-5175-3115.

\section{Appendix A. The finite-difference approximation to the boundary-region equations}

We denote the values of $\tilde{u}, \tilde{v}$ and $\tilde{\theta}$ at $\xi_{i}=(i-1) \Delta \xi, \Delta \xi=1 / N$ by $\tilde{u}\left(\xi_{i}\right)=\tilde{u}_{i}, \tilde{v}\left(\xi_{i}\right)=$ $\tilde{v}_{i}$ and $\tilde{\theta}\left(\xi_{i}\right)=\tilde{\theta}_{i}$, respectively, where $0 \leqslant i \leqslant N+1$. The wall is at $\xi_{1}=0$ and the production layer is at $\xi_{N}=(N-1) \Delta \xi=\xi_{P L}$.

The discretised boundary-region equations are

$$
\begin{gathered}
\alpha_{1} \tilde{u}_{i+1}+\alpha_{2} \tilde{u}_{i}+\alpha_{3} \tilde{u}_{i-1}=\alpha_{4} \tilde{v}_{i}+\alpha_{5} \tilde{\theta}_{i+1}+\alpha_{6} \tilde{\theta}_{i}+\alpha_{7} \tilde{\theta}_{i-1}, \\
\beta_{1} \tilde{v}_{i+2}+\beta_{2} \tilde{v}_{i+1}+\beta_{3} \tilde{v}_{i}+\beta_{4} \tilde{v}_{i-1}+\beta_{5} \tilde{v}_{i-2} \\
=\beta_{6} \tilde{\theta}_{i+2}+\beta_{7} \tilde{\theta}_{i+1}+\beta_{8} \tilde{\theta}_{i}+\beta_{9} \tilde{\theta}_{i-1}+\beta_{10} \tilde{\theta}_{i-2}, \\
\gamma_{1} \tilde{\theta}_{i+1}+\gamma_{2} \tilde{\theta}_{i}+\gamma_{3} \tilde{\theta}_{i-1}=\gamma_{4} \tilde{u}_{i+1}+\gamma_{5} \tilde{u}_{i-1}+\gamma_{6} \tilde{v}_{i} .
\end{gathered}
$$




\section{E.C. Johnstone and P. Hall}

The coefficients $\alpha_{k}, \beta_{k}$ and $\gamma_{k}$ depend on the coefficients $A_{k}, B_{k}$ and $C_{k}$. The coefficients of the finite-difference approximation to the $x$-momentum equation (6.19) are given by

$$
\begin{gathered}
\alpha_{1}=A_{2} / \Delta \xi+A_{3} / \Delta \xi^{2}, \quad \alpha_{2}=A_{1}-2 A_{3} / \Delta \xi^{2}, \quad \alpha_{3}=-A_{2} / \Delta \xi+A_{3} / \Delta \xi^{2}, \\
\alpha_{4}=A 4, \quad \alpha_{5}=A_{6} / 2 \Delta \xi, \quad \alpha_{6}=A_{5}, \quad \alpha_{7}=-A_{6} / 2 \Delta \xi .
\end{gathered}
$$

The coefficients of the finite-difference approximation to the $y$-momentum equation (6.20) are given by

$$
\begin{gathered}
\beta_{1}=B 4 / 2 \Delta \xi^{3}+B 5 / \Delta \xi^{4}, \\
\beta_{2}=B 2 / 2 \Delta \xi+B 3 / \Delta \xi^{2}-2 B 4 / 2 \Delta \xi^{3}-4 B 5 / \Delta \xi^{4}, \\
\beta_{3}=B 1-2 B 3 / \Delta \xi^{2}+6 B 5 / \Delta \xi^{4}, \\
\beta_{4}=-B 2 / 2 \Delta \xi+B 3 / \Delta \xi^{2}+2 B 4 / 2 \Delta \xi^{3}-4 B 5 / \Delta \xi^{4}, \\
\beta_{5}=-B 4 / 2 \Delta \xi^{3}+B 5 / \Delta \xi^{4}, \quad \beta_{6}=B 9 / 2 \Delta \xi^{3}+B 10 / \Delta \xi^{4}, \\
\beta_{7}=B 7 / 2 \Delta \xi+B 8 / \Delta \xi^{2}-2 B 9 / 2 \Delta \xi^{3}-4 B 10 / \Delta \xi^{4}, \\
\beta_{8}=B 6-2 B 8 / \Delta \xi^{2}+6 B 10 / \Delta \xi^{4}, \\
\beta_{9}=-B 7 / 2 \Delta \xi+B 8 / \Delta \xi^{2}+2 B 9 / 2 \Delta \xi^{3}-4 B 10 / \Delta \xi^{4}, \\
\beta_{10}=-B 9 / 2 \Delta \xi^{3}+B 10 / \Delta \xi^{4} .
\end{gathered}
$$

The coefficients of the finite-difference approximation to the temperature equation $(6.21)$ are given by

$$
\begin{gathered}
\gamma_{1}=C 1 / 2 \Delta \xi, \quad \gamma_{2}=-C 1 / 2 \Delta \xi, \quad \gamma_{3}=C 2, \quad \gamma_{4}=C 4 / 2 \Delta \xi+C 5 / \Delta \xi^{2}, \\
\gamma_{5}=C 3-2 C 5 / \Delta \xi^{2}, \quad \gamma_{6}=-C 4 / 2 \Delta \xi+C 5 / \Delta \xi^{2} .
\end{gathered}
$$

The coefficients A1-A6, B1-B10 and C1-C5 are available from the authors on request.

These finite-difference approximations are then encoded in a $3 \times 3$ block matrix $A$ where each block is of size $(N+2)^{2}$. The first, second and third block rows contain the discretisations of the $x$-momentum, $y$-momentum and temperature equations respectively. To find the solution $\tilde{\boldsymbol{u}}=\left(\tilde{u}\left(\xi_{i}\right), \tilde{v}\left(\xi_{i}\right), \tilde{\theta}\left(\xi_{i}\right)\right)^{\mathrm{T}}$ for $0 \leqslant i \leqslant N+1$ we solve $A \tilde{\boldsymbol{u}}=\boldsymbol{b}$, where $b$ contains the values of the solution and its derivatives at the boundaries.

\section{REFERENCES}

Blackaby, N.D., Cowley, S.J. \& Hall, P. 1993 On the instability of hypersonic flow past a flat plate. J. Fluid Mech. 247, 369-416.

CEBeci, T. 2002 Convective Heat Transfer, vol. 2. Springer.

Chapman, D.R. \& Rubesin, M.W. 1949 Temperature and velocity profiles in the compressible laminar boundary layer with arbitrary distribution of surface temperature. J. Aero. Sci. 16 (9), 547-565.

Cowley, S. \& Hall, P. 1990 On the instability of hypersonic flow past a wedge. J. Fluid Mech 214, 17-42.

DEGUCHI, K. 2017 Scaling of small vortices in stably stratified shear flows. J. Fluid Mech 821, 582-594.

Deguchi, K. \& HALl, P. 2014 Free-stream coherent structures in parallel boundary-layer flows. J. Fluid Mech. 752, 602-625.

DeGUCHI, K. \& HALL, P. 2015 Free-stream coherent structures in growing boundary layers: a link to near-wall streaks. J. Fluid Mech. 778, 451-484.

Deguchi, K. \& HALL, P. 2018 Free-stream coherent structures in a planar jet. J. Fluid Mech. 837, 916-930.

Demetriades, A. 1989 Growth of disturbances in a laminar boundary layer at Mach 3. Phys. Fluids A 1 (2), $312-317$. 


\section{Free-stream coherent structures in compressible shear flows}

Dempsey, L.J., Hall, P. \& Deguchi, K. 2017 The excitation of Görtler vortices by free-stream coherent structures. J. Fluid Mech. 826, 60-96.

Dorodnitsyn, A.A. 1942 Boundary layer in a compressible gas. Prikl. Mat. Mekh. 6 (6), 449-486.

EAVES, T.S. \& CAUlFIELD, C.P. 2015 Disruption of SSP/VWI states by a stable stratification. J. Fluid Mech. 784, 548-564.

FAisst, H. \& ECKHARDT, B. 2003 Traveling waves in pipe flow. Phys. Rev. Lett. 91 (22), 224502.

Ferro, M., Fallenius, B.E.G. \& Fransson, J.H.M. 2021 Experimental study on turbulent asymptotic suction boundary layers. J. Fluid Mech. 915, A80.

Fransson, J.H.M. \& Alfredsson, P.H. 2003 On the disturbance growth in an asymptotic suction boundary layer. J. Fluid Mech. 482, 51-90.

Fransson, J.H.M., Matsubara, M. \& Alfredsson, P.H. 2005 Transition induced by free-stream turbulence. J. Fluid Mech. 527, 1-25.

FRANSSON, J.H.M. \& ShahinfAR, S. 2020 On the effect of free-stream turbulence on boundary-layer transition. J. Fluid Mech. 899, A23.

Fu, Y., HALL, P. \& BlackABY, N. 1993 On the Görtler instability in hypersonic flows: Sutherland law fluids and real gas effects. Phil. Trans. R. Soc. Lond. A 342 (1665), 325-377.

Graziosi, P. \& Brown, G.L. 2002 Experiments on stability and transition at Mach 3. J. Fluid Mech. 472, 83-124.

Hall, P. 1983 The linear development of Görtler vortices in growing boundary layers. J. Fluid Mech. 130, 41-58.

HALl, P. \& Sherwin, S. 2010 Streamwise vortices in shear flows: harbingers of transition and the skeleton of coherent structures. J. Fluid Mech. 661, 178-205.

HALL, P. \& SMith, F.T. 1991 On strongly nonlinear vortex/wave interactions in boundary-layer transition. J. Fluid Mech. 227, 641-666.

Hocking, L.M. 1975 Non-linear instability of the asymptotic suction velocity profile. Q. J. Mech. Appl. Maths 28 (3), 341-353.

HOWARTH, L. 1948 Concerning the effect of compressibility on laminar boundary layers and their separation. Proc. R. Soc. Lond. A 194 (1036), 16-42.

INTERNATIONAL ORGANIZATION FOR STANDARDIZATION 1975 Standard Atmosphere. ISO 2533:1975.

Johnstone, E.C. \& HALL, P. 2020 Free-stream coherent structures in the unsteady Rayleigh boundary layer. IMA J. Appl. Maths 85 (6), 1021-1040.

KENDALL, J.M. 1975 Wind tunnel experiments relating to supersonic and hypersonic boundary-layer transition. AIAA J. 13 (3), 290-299.

Khapko, T., Kreilos, T., Schlatter, P., Duguet, Y., Eckhardt, B. \& Henningson, D.S. 2013 Localized edge states in the asymptotic suction boundary layer. J. Fluid Mech. 717, R6.

Kline, S.J., Reynolds, W.C., Schraub, F.A. \& Runstadler, P.W. 1967 The structure of turbulent boundary layers. J. Fluid Mech. 30 (4), 741-773.

Kreilos, T., Gibson, J.F. \& SCHNEIDER, T.M. 2016 Localized travelling waves in the asymptotic suction boundary layer. J. Fluid Mech. 795, R3.

Kreilos, T., Veble, G., Schneider, T.M. \& EckhardT, B. 2013 Edge states for the turbulence transition in the asymptotic suction boundary layer. J. Fluid Mech. 726, 100-122.

Langham, J., EAves, T.S. \& Kerswell, R.R. 2020 Stably stratified exact coherent structures in shear flow: the effect of Prandtl number. J. Fluid Mech. 882, A10.

LAUFER, J. 1954 Factors affecting transition Reynolds numbers on models in supersonic wind tunnels. J. Aero. Sci. 21 (7), 497-498.

Leib, S.J., Wundrow, D.W. \& Goldstein, M.E. 1999 Effect of free-stream turbulence and other vortical disturbances on a laminar boundary layer. J. Fluid Mech. 380 (1), 169-203.

LUCAS, D. \& CAULFIELD, C.P. 2017 Irreversible mixing by unstable periodic orbits in buoyancy dominated stratified turbulence. J. Fluid Mech. 832, R1.

LuCAS, D., CAulfield, C.P. \& Kerswell, R.R. 2017 Layer formation in horizontally forced stratified turbulence: connecting exact coherent structures to linear instabilities. J. Fluid Mech. 832, 409-437.

MACK, L.M. 1975 Linear stability theory and the problem of supersonic boundary-layer transition. AIAA J. 13 (3), 278-289.

Mack, L.M. 1984 Boundary-layer linear stability theory. In Special Course on Stability and Transition of Laminar Flow, AGARD Rep. 709. Jet Propulsion Laboratory.

Marensi, E., RiCCO, P. \& WU, X. 2017 Nonlinear unsteady streaks engendered by the interaction of free-stream vorticity with a compressible boundary layer. J. Fluid Mech. 817, 80-121.

MAYER, C.S.J., VON TERZI, D.A. \& FASEL, H.F. 2011 Direct numerical simulation of complete transition to turbulence via oblique breakdown at Mach 3. J. Fluid Mech. 674, 5-42. 


\section{E.C. Johnstone and P. Hall}

Morkovin, M.V. 1962 Effects of compressibility on turbulent flows. In Mécanique de la Turbulence (ed. A. Favre), pp. 367-380. CNRS.

Olvera, D. \& Kerswell, R.R. 2017 Exact coherent structures in stably stratified plane Couette flow. J. Fluid Mech. 826, 583-614.

Pirozzoli, S., Bernardini, M. \& Grasso, F. 2008 Characterization of coherent vortical structures in a supersonic turbulent boundary layer. J. Fluid Mech. 613, 205-231.

RicCO, P. \& WU, X. 2007 Response of a compressible laminar boundary layer to free-stream vortical disturbances. J. Fluid Mech. 587, 97-138.

Ringuette, M.J., Wu, M. \& Martín, M.P. 2008 Coherent structures in direct numerical simulation of turbulent boundary layers at Mach 3. J. Fluid Mech. 594, 59-69.

Schlichting, H. 1968 Boundary Layer Theory, vol. 960. Springer.

SpinA, E.F., Smits, A.J. \& Robinson, S.K. 1994 The physics of supersonic turbulent boundary layers. Annu. Rev. Fluid Mech. 26 (1), 287-319.

Sutherland, W. 1893 LII. The viscosity of gases and molecular force. Lond. Edin. Dublin Phil. Mag. J. Sci. 36 (223), 507-531.

WALEFFE, F. 1997 On a self-sustaining process in shear flows. Phys. Fluids 9 (4), 883-900.

WALEFFE, F. 2001 Exact coherent structures in channel flow. J. Fluid Mech. 435, 93-102.

WALEFFE, F. 2003 Homotopy of exact coherent structures in plane shear flows. Phys. Fluids 15 (6), 1517-1534.

WAng, J., Gibson, J. \& WalefFe, F. 2007 Lower branch coherent states in shear flows: transition and control. Phys. Rev. Lett. 98 (20), 204501.

WEDIN, H. \& KERSWELL, R.R. 2004 Exact coherent structures in pipe flow: travelling wave solutions. J. Fluid Mech. 508, 333-371.

Wu, X. \& DONG, M. 2016 Entrainment of short-wavelength free-stream vortical disturbances in compressible and incompressible boundary layers. J. Fluid Mech. 797, 683-728. 